FedUni ResearchOnline

http://researchonline.federation.edu.au

This is the author's accepted version of the following publication:

Kruger, A. (2015) Error bounds and Hölder metric subregularity.

Set-Valued and Variational Analysis, 23(4), 705-736.

The version displayed here may differ from the final published version.

The final publication is available at:

http://doi.org/10.1007/s11228-015-0330-y

Copyright (C) 2015, Springer Science+Business Media B.V. 


\title{
Error Bounds and Hölder Metric Subregularity
}

\author{
Alexander Y. Kruger \\ Dedicated to Professor Lionel Thibault
}

Received: date / Accepted: date

\begin{abstract}
The Hölder setting of the metric subregularity property of set-valued mappings between general metric or Banach/Asplund spaces is investigated in the framework of the theory of error bounds for extended real-valued functions of two variables. A classification scheme for the general Hölder metric subregularity criteria is presented. The criteria are formulated in terms of several kinds of primal and subdifferential slopes.
\end{abstract}

Keywords error bounds; slope; metric regularity; metric subregularity; Hölder metric subregularity

Mathematics Subject Classification (2000) 49J52; 49J53; 58C06; 47H04; 54C60

\section{Introduction}

In this paper, we investigate the Hölder setting of the metric subregularity property of set-valued mappings between general metric or Banach/Asplund spaces in the framework of the theory of error bounds for extended real-valued functions of two variables developed in [31]. Several primal and dual space local quantitative and qualitative criteria of Hölder metric subregularity are formulated. The relationships between the criteria are established and illustrated.

Recall that an extended-real-valued function $f: X \rightarrow \mathbb{R}_{\infty}:=\mathbb{R} \cup\{+\infty\}$ on a metric space $X$ is said to have a local error bound (cf., e.g., $[2,4,13,22]$ ) with constant $\tau>0$ at a point $\bar{x} \in X$ with $f(\bar{x})=0$ if there exists a neighbourhood $U$ of $\bar{x}$ such that

$$
\tau d(x, S(f)) \leq f_{+}(x) \quad \text { for all } x \in U \text {. }
$$

Here $S(f)$ stands for the lower 0-level set $\{x \in X \mid f(x) \leq 0\}$ and $f_{+}(x):=\max \{f(x), 0\}$.

The research was supported by the Australian Research Council, project DP110102011.

A. Y. Kruger

Centre for Informatics and Applied Optimization, Faculty of Science and Technology, Federation University Australia, POB 663, Ballarat, Vic, 3350, Australia

E-mail: a.kruger@federation.edu.au 
A set-valued mapping $F: X \rightrightarrows Y$ is a mapping which assigns to every $x \in X$ a subset (possibly empty) $F(x)$ of $Y$. We use the notation

$$
\operatorname{gph} F:=\{(x, y) \in X \times Y \mid y \in F(x)\}
$$

for the graph of $F$ and $F^{-1}: Y \rightrightarrows X$ for the inverse of $F$. This inverse (which always exists) is defined by

$$
F^{-1}(y):=\{x \in X \mid y \in F(x)\}, \quad y \in Y
$$

and satisfies

$$
(x, y) \in \operatorname{gph} F \quad \Leftrightarrow \quad(y, x) \in \operatorname{gph} F^{-1} .
$$

A set-valued mapping $F: X \rightrightarrows Y$ between metric spaces is called (locally) metri-

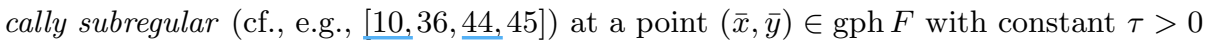
if there exist neighbourhoods $U$ of $\bar{x}$ and $V$ of $\bar{y}$ such that

$$
\tau d\left(x, F^{-1}(\bar{y})\right) \leq d(\bar{y}, F(x) \cap V) \quad \text { for all } x \in U .
$$

This property represents a weaker version of the more robust metric regularity property which corresponds to replacing $\bar{y}$ in the above inequality by an arbitrary (not fixed!) $y \in V$.

It is well known that one can always take $V=Y$ in the definition of metric subregularity, which is, thus, equivalent to the existence of a neighbourhood $U$ of $\bar{x}$ such that

$$
\tau d\left(x, F^{-1}(\bar{y})\right) \leq d(\bar{y}, F(x)) \quad \text { for all } x \in U .
$$

The metric subregularity (and closely related to it calmness) property plays an important role in both theory and applications. The amount of publications devoted to (mostly sufficient) criteria of metric subregularity is huge. The interested reader is referred to $[1,9,10,19-21,26,33,43,48-51]$.

In many important situations, the standard metric (sub)regularity property is not satisfied, and more subtle Hölder type estimates come into play.

If instead of (1) one uses the following more general condition:

$$
\tau d\left(x, F^{-1}(\bar{y})\right) \leq(d(\bar{y}, F(x)))^{q} \text { for all } x \in U,
$$

where $q \in(0,1]$, then the corresponding property is usually referred to as Hölder metric subregularity of order $q$ at $(\bar{x}, \bar{y})$ with constant $\tau$. The case $q=1$ corresponds to standard metric subregularity. If $q_{1}<q_{2} \leq 1$, then Hölder metric subregularity of order $q_{2}$ implies that of order $q_{2}$.

If fixed $\bar{y}$ in the above inequality is replaced by an arbitrary $y$ and the inequality is required to hold uniformly over all $y$ near $\bar{y}$, then we arrive at the definition of Hölder metric regularity of order $q$. The latter property and even more general nonlinear regularity models have been studied since 1980s; cf. [6, 15-17, 25, 42, 46].

The history of the Hölder metric subregularity property seems to be significantly shorter with most work done in the last few years; cf. [18, 28,29,32,34,37,40]. Note the only attempt so far to consider the case $q>1$ in [37].

One can easily see that Hölder metric subregularity property (2) is equivalent to the local error bound property of the extended real-valued function $x \mapsto(d(\bar{y}, F(x)))^{q}$ at $\bar{x}$ (with the same constant). So one might want to apply to this model the well

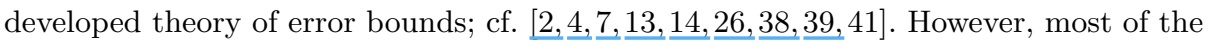


error bound criteria are formulated for lower semicontinuous functions (cf. Section 2), while the function $x \mapsto(d(\bar{y}, F(x)))^{q}$ can fail to be lower semicontinuous even when gph $F$ is closed.

Another helpful observation is that property (2) can be rewritten equivalently as

$$
\tau d\left(x, F^{-1}(\bar{y})\right) \leq(d(y, \bar{y}))^{q} \text { for all } x \in U, y \in F(x) .
$$

This allows one to apply to it the extension of the theory of error bounds to functions of two variables developed in [31] and used there for characterizing standard metric subregularity (case $q=1$ ).

Following the standard trend initiated by Ioffe [24], criteria for error bounds and Hölder metric subregularity of set-valued mappings in metric spaces are formulated in terms of (strong) slopes [8]. To simplify the statements in metric and also Banach/Asplund spaces, several other kinds of primal and dual space slopes for real-valued functions and set-valued mappings are discussed in this article and the relationships between them are formulated. These relationships lead to a simple hierarchy of the error bound and Hölder metric subregularity criteria.

Some statements in the article look rather long because each of them contains an almost complete list of criteria applicable in the situation under consideration. The reader is not expected to read through the whole list. Instead, they can select a particular criterion or a group of criteria corresponding to the setting of interest to them (e.g., in metric or Banach/Asplund/smooth spaces, in the convex case, etc.)

Our basic notation is standard, cf. $[10,36,44,45]$. Depending on the context, $X$ and $Y$ are either metric or normed spaces. Metrics in all spaces are denoted by the same symbol $d(\cdot, \cdot) ; d(x, A):=\inf _{a \in A} d(x, a)$ is the point-to-set distance from $x$ to $A$. $B_{\delta}(x)$ denotes the closed ball with radius $\delta$ and centre $x$. If not specified otherwise, the product of metric/normed spaces is assumed equipped with the distance/norm given by the maximum of the distances/norms.

If $X$ and $Y$ are normed spaces, their topological duals are denoted $X^{*}$ and $Y^{*}$, respectively, while $\langle\cdot, \cdot\rangle$ denotes the bilinear form defining the pairing between the spaces. The closed unit balls in a normed space and its dual are denoted by $\mathbb{B}$ and $\mathbb{B}^{*}$, respectively, while $\mathbb{S}$ and $\mathbb{S}^{*}$ stand for the unit spheres.

We say that a subset $\Omega$ of a metric space is locally closed near $\bar{x} \in \Omega$ if $\Omega \cap U$ is closed for some closed neighbourhood $U$ of $\bar{x}$. Given an $\alpha \in \mathbb{R}_{\infty}, \alpha_{+}$denotes its "positive" part: $\alpha_{+}:=\max \{\alpha, 0\}$.

If $X$ is a normed linear space, $f: X \rightarrow \mathbb{R}_{\infty}, x \in X$, and $f(x)<\infty$, then

$$
\partial f(x):=\left\{x^{*} \in X^{*} \mid \liminf _{u \rightarrow x, u \neq x} \frac{f(u)-f(x)-\left\langle x^{*}, u-x\right\rangle}{\|u-x\|} \geq 0\right\}
$$

is the Fréchet subdifferential of $f$ at $x$. Similarly, if $x \in \Omega \subset X$, then

$$
N_{\Omega}(x):=\left\{x^{*} \in X^{*} \mid \limsup _{u \rightarrow x, u \in \Omega \backslash\{x\}} \frac{\left\langle x^{*}, u-x\right\rangle}{\|u-x\|} \leq 0\right\}
$$

is the Fréchet normal cone to $\Omega$ at $x$. In the convex case, sets (3) and (4) reduce to the subdifferential and normal cone in the sense of convex analysis, respectively. If $f(x)=\infty$ or $x \notin \Omega$, we set, respectively, $\partial f(x)=\emptyset$ or $N_{\Omega}(x)=\emptyset$. Observe that definitions (3) and (4) are invariant on the renorming of the space (replacing the norm by an equivalent one). 
If $F: X \rightrightarrows Y$ is a set-valued mapping between normed linear spaces and $(x, y) \in$ $\operatorname{gph} F$, then

$$
D^{*} F(x, y)\left(y^{*}\right):=\left\{x^{*} \in X^{*} \mid\left(x^{*},-y^{*}\right) \in N_{\operatorname{gph} F}(x, y)\right\}, \quad y^{*} \in X^{*}
$$

is the Fréchet coderivative of $F$ at $(x, y)$.

Several kinds of subdifferential sum rules are employed in the article. Below we provide these rules for completeness.

Lemma 1 (Subdifferential sum rules) Suppose $X$ is a normed linear space, $f_{1}, f_{2}$ : $X \rightarrow \mathbb{R}_{\infty}$, and $\bar{x} \in \operatorname{dom} f_{1} \cap \operatorname{dom} f_{2}$.

(i) Fuzzy sum rule. Suppose $X$ is Asplund, $f_{1}$ is Lipschitz continuous and $f_{2}$ is lower semicontinuous in a neighbourhood of $\bar{x}$. Then, for any $\varepsilon>0$, there exist $x_{1}, x_{2} \in X$ with $\left\|x_{i}-\bar{x}\right\|<\varepsilon,\left|f_{i}\left(x_{i}\right)-f_{i}(\bar{x})\right|<\varepsilon(i=1,2)$ such that

$$
\partial\left(f_{1}+f_{2}\right)(\bar{x}) \subset \partial f_{1}\left(x_{1}\right)+\partial f_{2}\left(x_{2}\right)+\varepsilon \mathbb{B}^{*} .
$$

(ii) Differentiable sum rule. Suppose $f_{1}$ is Fréchet differentiable at $\bar{x}$. Then,

$$
\partial\left(f_{1}+f_{2}\right)(\bar{x})=\nabla f_{1}(\bar{x})+\partial f_{2}(\bar{x})
$$

(iii) Convex sum rule. Suppose $f_{1}$ and $f_{2}$ are convex and $f_{1}$ is continuous at a point in $\operatorname{dom} f_{2}$. Then,

$$
\partial\left(f_{1}+f_{2}\right)(\bar{x})=\partial f_{1}(\bar{x})+\partial f_{2}(\bar{x}) .
$$

The first sum rule in the lemma above is known as the fuzzy or approximate sum rule (Fabian [12]; cf., e.g., [30, Rule 2.2], [36, Theorem 2.33]) for Fréchet subdifferentials in Asplund spaces. The other two are examples of exact sum rules. They are valid in arbitrary normed spaces (or even locally convex spaces in the case of the last rule). Rule (ii) can be found, e.g., in [30, Corollary 1.12.2] and [36, Proposition 1.107]. For rule (iii) we refer the readers to [27, Theorem 0.3.3] and [47, Theorem 2.8.7].

Recall that a Banach space is Asplund if the dual of each its separable subspace is separable; see, e.g., $[5,36]$ for discussions and characterizations of Asplund spaces. Note that any Fréchet smooth space, i.e., a Banach space which admits an equivalent norm, Fréchet differentiable at all nonzero points, is Asplund. Given a Fréchet smooth space, we will always assume that it is endowed with such a norm.

The (normalized) duality mapping $J$ between a normed space $Y$ and its dual $Y^{*}$ is defined as (cf. [35, Definition 3.2.6])

$$
J(y):=\left\{y^{*} \in \mathbb{S}_{Y^{*}} \mid\left\langle y^{*}, y\right\rangle=\|y\|\right\}, \quad y \in Y .
$$

Note that $J(-y)=-J(y)$. The following simple fact of convex analysis is well known (cf., e.g., [47, Corollary 2.4.16]).

Lemma 2 Let $(Y,\|\cdot\|)$ be a normed space.

(i) $\partial\|\cdot\|(y)=J(y)$ for any $y \neq 0$.

(ii) $\partial\|\cdot\|(0)=\mathbb{B}^{*}$. 
The structure of the article is as follows. In the next section, we present a survey and some extensions of the error bound criteria for a special family of extended-realvalued functions on products of either metric or Banach/Asplund spaces. The criteria are formulated in terms of several kinds of primal and subdifferential slopes. The relationships between the slopes are presented. In Section 3, we demonstrate how Hölder metric subregularity of set-valued mappings can be treated in the framework of the theory of error bounds. A collection of slopes for a set-valued mapping are discussed. The final Section 4 is dedicated to primal and subdifferential criteria for Hölder metric subregularity.

\section{Error bounds and slopes}

In this section, we discuss a general model of error bounds for an extended-real-valued function $f: X \times Y \rightarrow \mathbb{R}_{\infty}$ on a product of metric spaces considered in [31] and recall several error bound criteria in terms of (several kinds of) slopes.

It is assumed that $f(\bar{x}, \bar{y})=0$, and $f$ depends on its second variable in a special way:

(P1) $f(x, y)>0$ if $y \neq \bar{y}$

(P2) $\liminf _{f(x, y) \downarrow 0} \frac{f(x, y)}{d(y, \bar{y})}>0$.

In particular, condition $f(x, y) \downarrow 0$ implies that $y \rightarrow \bar{y}$.

Function $f$ is said to have an error bound with respect to $x$ at $(\bar{x}, \bar{y})$ with constant $\tau>0$ if there exists a neighbourhood $U$ of $\bar{x}$ such that

$$
\tau d(x, S(f)) \leq f_{+}(x, y) \text { for all } x \in U, y \in Y,
$$

where

$$
S(f):=\{x \in X \mid f(x, \bar{y}) \leq 0\}=\{x \in X \mid f(x, y) \leq 0 \text { for some } y \in Y\} .
$$

The second equality is a consequence of (P1).

The error bound modulus

$$
\operatorname{Er} f(\bar{x}, \bar{y}):=\liminf _{\substack{x \rightarrow \bar{x} \\ f(x, y)>0}} \frac{f(x, y)}{d(x, S(f))}
$$

coincides with the exact upper bound of all $\tau>0$ such that (6) holds true for some neighbourhood $U$ of $\bar{x}$ and provides a quantitative characterization of the error bound property.

Observe that the case of a function $f: X \rightarrow \mathbb{R}_{\infty}$ of a single variable can be covered by considering its extension $\tilde{f}: X \times Y \rightarrow \mathbb{R}_{\infty}$ defined, for some $\bar{y} \in Y$, by

$$
\tilde{f}(x, y)= \begin{cases}f(x) & \text { if } y=\bar{y} \\ \infty & \text { otherwise }\end{cases}
$$

Conditions (P1) and (P2) are obviously satisfied.

Definition (7) admits the following equivalent representations.

Proposition $1 \operatorname{Er} f(\bar{x}, \bar{y})=\liminf _{\substack{x \rightarrow \bar{x}, y \rightarrow \bar{y} \\ f(x, y)>0}} \frac{f(x, y)}{d(x, S(f))}=\liminf _{x \rightarrow \bar{x}, f(x, y) \downarrow 0} \frac{f(x, y)}{d(x, S(f))}$. 
The roles of variables $x$ and $y$ in definitions (6) and (7) are different. To better reflect this, we consider the following asymmetric maximum-type distance in $X \times Y$ depending on a positive parameter $\rho$ :

$$
d_{\rho}((x, y),(u, v)):=\max \{d(x, u), \rho d(y, v)\} .
$$

Alternatively, one can use the parametric sum-type distance:

$$
d_{\rho}^{1}((x, y),(u, v)):=d(x, u)+\rho d(y, v) .
$$

To formulate (nonlocal) primal space characterizations of the error bound property (6), we are going to use the following two (possibly infinite) constants:

$$
\begin{gathered}
|\nabla f|_{\rho}^{\diamond}(x, y):=\sup _{(u, v) \neq(x, y)} \frac{\left[f(x, y)-f_{+}(u, v)\right]_{+}}{d_{\rho}((x, y),(u, v))}, \\
|\nabla f|^{\diamond}(\bar{x}, \bar{y}):=\lim _{\rho \downarrow 0 d(x, \bar{x})<\rho, 0<f(x, y)<\rho} \inf |\nabla f|_{\rho}^{\diamond}(x, y),
\end{gathered}
$$

which are called, respectively, the nonlocal $\rho$-slope of $f$ at $(x, y)$ and the uniform strict outer slope. It is assumed in $(10)$ that $f(x, y)<\infty$. If $f(x, y)=\infty$, we set $|\nabla f|_{\rho}^{\diamond}(x, y)=$ $\infty$. In (11), the usual convention that the infimum of the empty set equals $+\infty$ is in force.

The word "strict" reflects the fact that $\rho$-slopes at nearby points contribute to definition (11) making it an analogue of the strict derivative. The word "outer" is used to emphasize that only points outside the set $S(f)$ are taken into account. The word "uniform" emphasizes the nonlocal (non-limiting) character of $|\nabla f|_{\rho}^{\diamond}(x, y)$ involved in definition (11).

In the sequel, superscript ' $\diamond$ ' (diamond) will be used in all constructions derived from (10) and its analogues to distinguish them from "conventional" (local) definitions.

Definition (10) is a realization of the nonlocal slope [14] for the case of a function on a product space with the product metric defined by (8). In definition (11), we have not only $x \rightarrow \bar{x}$ and $f(x, y) \downarrow 0$, but also the metric on $X \times Y$ used in the definition of the nonlocal $\rho$-slope $|\nabla f|_{\rho}^{\diamond}(x, y)$ changing with the contribution of the $y$ component diminishing as $\rho \downarrow 0$.

The local analogues of (10) and (11) are defined as follows:

$$
\begin{gathered}
|\nabla f|_{\rho}(x, y):=\limsup _{\substack{u \rightarrow x, v \rightarrow y \\
(u, v) \neq(x, y)}} \frac{[f(x, y)-f(u, v)]_{+}}{d_{\rho}((u, v),(x, y))}, \\
\overline{|\nabla f|}^{>}(\bar{x}, \bar{y}):=\lim _{\rho \downarrow 0 d(x, \bar{x})<\rho, 0<f(x, y)<\rho}|\nabla f|_{\rho}(x, y)
\end{gathered}
$$

and are called, respectively, the $\rho$-slope of $f$ at $(x, y)(f(x, y)<\infty)$ and the strict outer slope of $f$ at $(\bar{x}, \bar{y})$. Definition (12) is a realization of the local (strong) slope [8] for the case of a function on a product space with the product metric defined by (8).

The next modified strict outer slope of $f$ at $(\bar{x}, \bar{y})$ can be useful when estimating the error bound modulus:

$$
\overline{|\nabla f|}^{>+}(\bar{x}, \bar{y}):=\lim _{\rho \downarrow 0} \inf _{d(x, \bar{x})<\rho, 0<f(x, y)<\rho} \max \left\{|\nabla f|_{\rho}(x, y), \frac{f(x, y)}{d(x, \bar{x})}\right\} .
$$

Observe that, unlike the completely local constant (13), its modified counterpart (14) contains under max a nonlocal (when $(x, y)$ is fixed) component $f(x, y) / d(x, \bar{x})$. 
In normed linear spaces, one can use for estimating slopes and hence error bounds other tools based on either directional derivatives or subdifferentials. Below we describe some tools from the second group.

Suppose $X$ and $Y$ are normed linear spaces. In the product space $X \times Y$, along with the usual maximum-type norm

$$
\|(u, v)\|=\max \{\|u\|,\|v\|\}, \quad(u, v) \in X \times Y,
$$

we consider the $\rho$-norm $\|\cdot\|_{\rho}$ being the realization of the $\rho$-metric (8):

$$
\|(u, v)\|_{\rho}=\max \{\|u\|, \rho\|v\|\}, \quad(u, v) \in X \times Y .
$$

The corresponding dual norm (we keep the same notation $\|\cdot\|_{\rho}$ for it) is of the form:

$$
\left\|\left(u^{*}, v^{*}\right)\right\|_{\rho}=\left\|u^{*}\right\|+\rho^{-1}\left\|v^{*}\right\|, \quad\left(u^{*}, v^{*}\right) \in X^{*} \times Y^{*} .
$$

One can define subdifferential counterparts of the local slopes (12)-(14): the subdifferential $\rho$-slope

$$
|\partial f|_{\rho}(x, y):=\inf _{\left(x^{*}, y^{*}\right) \in \partial f(x, y),\left\|y^{*}\right\|<\rho}\left\|x^{*}\right\|
$$

of $f$ at $(x, y)(f(x, y)<\infty)$ and the strict outer and, respectively, modified strict outer subdifferential slopes

$$
\begin{aligned}
& \overline{|\partial f|}^{>}(\bar{x}, \bar{y}):=\lim _{\rho \downarrow 0} \inf _{\|x-\bar{x}\|<\rho, 0<f(x, y)<\rho}|\partial f|_{\rho}(x, y), \\
& \overline{|\partial f|}^{>+}(\bar{x}, \bar{y}):=\lim _{\rho \downarrow 0} \inf _{\|x-\bar{x}\|<\rho, 0<f(x, y)<\rho} \max \left\{|\partial f|_{\rho}(x, y), \frac{f(x, y)}{\|x-\bar{x}\|}\right\}
\end{aligned}
$$

of $f$ at $(\bar{x}, \bar{y})$.

Remark 1 Definitions (11), (13), (14), (16), and (17) corresponding to the lower 0level set $S(f)$ of $f$ can be easily extended to the case of the general lower level set $\{x \in X \mid f(x, \bar{y}) \leq f(\bar{x}, \bar{y})\}$ with an arbitrary finite $f(\bar{x}, \bar{y})$. It is sufficient to replace $f$ in (P1), (P2), (10), (11), (13), (14), (16), and (17) with function $(x, y) \mapsto f(x, y)-f(\bar{x}, \bar{y})$.

Remark $2 \rho$-slopes (10), (12), and (15) are nondecreasing functions of $\rho$. This makes the infimums in (11), (13), (14), (16), and (17) nondecreasing functions of $\rho$ too. This observation allows one to simplify estimates involving strict slopes, e.g., in Corollary 1 below.

The next proposition summarizes the relationships between the slopes. It extends [31, Proposition 3.2 and Theorem 3.3].

\section{Proposition 2 (Relationships between slopes)}

(i) $|\nabla f|_{\rho}^{\diamond}(x, y) \geq \max \left\{|\nabla f|_{\rho}(x, y), \frac{f(x, y)}{d_{\rho}((x, y),(\bar{x}, \bar{y}))}\right\}$

for all $\rho>0$ and all $(x, y) \in X \times Y$ with $0<f(x, y)<\infty$;

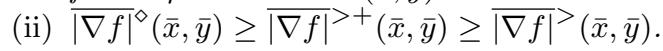

Suppose $X$ and $Y$ are normed linear spaces.

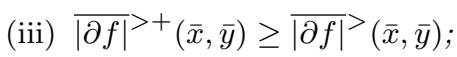


(iv) $|\nabla f|_{\rho}(x, y) \leq|\partial f|_{\rho^{2}}(x, y)+\rho$ for all $\rho>0$ and all $(x, y) \in X \times Y$ with $f(x, y)<\infty$;

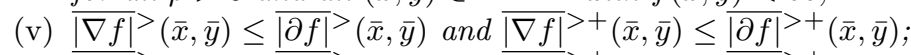

(vi) $|\overline{\nabla f \mid}|^{>}(\bar{x}, \bar{y})=\left.\overline{|\partial f|}\right|^{>}(\bar{x}, \bar{y})$ and $|\overline{\nabla f}|^{>+}(\bar{x}, \bar{y})=|\overline{\mid \partial f}|^{>+}(\bar{x}, \bar{y})$

provided that one of the following conditions is satisfied:

(a) $X$ and $Y$ are Asplund and $f_{+}$is lower semicontinuous near $(\bar{x}, \bar{y})$;

(b) $f$ is convex near $(\bar{x}, \bar{y})$;

(c) $f$ is Fréchet differentiable near $(\bar{x}, \bar{y})$ except $(\bar{x}, \bar{y})$;

(d) $f=f_{1}+f_{2}$, where $f_{1}$ is convex near $(\bar{x}, \bar{y})$ and $f_{2}$ is Fréchet differentiable near $(\bar{x}, \bar{y})$ except $(\bar{x}, \bar{y})$

(vii) if $f$ is convex near $(\bar{x}, \bar{y})$, then

(a) $|\nabla f|_{\rho}^{\diamond}(x, y)=|\nabla f|_{\rho}(x, y)$

for all $\rho>0$ and all $(x, y) \in X \times Y$ near $(\bar{x}, \bar{y})$ with $0<f(x, y)<\infty$;

(b) $|\overline{\nabla f}|^{\diamond}(\bar{x}, \bar{y})=\left.\overline{\mid \nabla f}\right|^{>+}(\bar{x}, \bar{y})=\left.\overline{\mid \nabla f}\right|^{>}(\bar{x}, \bar{y})=\left.\overline{\mid \partial f}\right|^{>+}(\bar{x}, \bar{y})=|\overline{\partial f}|^{>}(\bar{x}, \bar{y})$.

Proof (i) Inequality $|\nabla f|_{\rho}^{\diamond}(x, y) \geq|\nabla f|_{\rho}(x, y)$ comes from [31, Proposition 3.2(i)], while the other inequality is a direct consequence of definition (10).

(ii) The second inequality follows from comparing definitions (13) and (14), while the first inequality requires a modification of the proof of [31, Proposition 3.2(iii)]. Let $\overline{|\nabla f|^{\diamond}}(\bar{x}, \bar{y})<\gamma<\infty$ and $\rho>0$. By (P2), one can find a $\rho^{\prime} \in(0, \rho)$ such that

$$
\frac{f(x, y)}{d(y, \bar{y})}>\rho^{\prime} \gamma
$$

as long as $0<f(x, y)<\rho^{\prime}$. By (11), there exists a point $(x, y) \in X \times Y$ with $d(x, \bar{x})<\rho^{\prime}$ and $0<f(x, y)<\rho^{\prime}$ such that $|\nabla f|_{\rho^{\prime}}^{\diamond}(x, y)<\gamma$, i.e., by $(10)$,

$$
\frac{f(x, y)-f_{+}(u, v)}{d_{\rho^{\prime}}((x, y),(u, v))}<\gamma
$$

for all $(u, v) \neq(x, y)$. Observe that $(x, y) \neq(\bar{x}, \bar{y})$ since $f(x, y)>0$. Hence, $|\nabla f|_{\rho}(x, y) \leq$ $\gamma$ and

$$
\frac{f(x, y)}{d_{\rho^{\prime}}((x, y),(\bar{x}, \bar{y}))}=\min \left\{\frac{f(x, y)}{d(x, \bar{x})},\left(\rho^{\prime}\right)^{-1} \frac{f(x, y)}{d(y, \bar{y})}\right\}<\gamma .
$$

Together with (18), this implies $f(x, y) / d(x, \bar{x})<\gamma$. Thus,

$$
\max \left\{|\nabla f|_{\rho}(x, y), \frac{f(x, y)}{d(x, \bar{x})}\right\} \leq \gamma
$$

and consequently,

$$
\inf _{d(x, \bar{x})<\rho, 0<f(x, y)<\rho} \max \left\{|\nabla f|_{\rho}(x, y), \frac{f(x, y)}{d(x, \bar{x})}\right\} \leq \gamma .
$$

Taking limits as $\rho \downarrow 0$ and $\gamma \downarrow \overline{|\nabla f|}^{\diamond}(\bar{x}, \bar{y})$, we arrive at the claimed inequality.

(iii) is a consequence of definitions (16) and (17).

(iv) comes from [31, Theorem 3.3(i)].

(v) is a consequence of (v) and definitions (16) and (17).

(vi) Under condition (a), the first equality comes from [31, Theorem 3.3(iii)], while the second one requires a modification of the proof of that theorem.

Let $X$ and $Y$ be Asplund and $f_{+}$be lower semicontinuous near $(\bar{x}, \bar{y})$. Thanks to (v), we only need to prove that $\overline{|\nabla f|}^{>+}(\bar{x}, \bar{y}) \geq \overline{\mid \partial f}^{>+}(\bar{x}, \bar{y})$. If $\overline{|\nabla f|}^{>+}(\bar{x}, \bar{y})=\infty$, the 
assertion is trivial. Let $\left.\overline{|\nabla f|}^{>+}{ }_{(\bar{x}}, \bar{y}\right)<\gamma<\infty$. Choose a $\left.\gamma^{\prime} \in\left(\overline{|\nabla f|}{ }^{+}{ }_{(\bar{x}}, \bar{y}\right), \gamma\right)$ and an arbitrary $\rho>0$. Set $\rho^{\prime}:=\min \left\{1, \gamma^{-1}\right\} \rho$. By definitions (14) and (12), one can find a point $(x, y) \in X \times Y$ such that $d(x, \bar{x})<\rho^{\prime}, 0<f(x, y)<\rho^{\prime}, f(x, y) / d(x, \bar{x})<\gamma^{\prime}, f$ is lower semicontinuous near $(x, y)$, and

$$
f(x, y)-f(u, v) \leq \gamma^{\prime}\|(u, v)-(x, y)\|_{\rho^{\prime}} \quad \text { for all }(u, v) \text { near }(x, y) .
$$

In other words, $(x, y)$ is a point of local minimum of the function

$$
(u, v) \mapsto f(u, v)+\gamma^{\prime}\|(u, v)-(x, y)\|_{\rho^{\prime}} .
$$

Take an

$$
\varepsilon \in\left(0, \min \left\{\rho-d(x, \bar{x}), \rho-f(x, y), \gamma-\gamma^{\prime}, \frac{\gamma d(x, \bar{x})-f(x, y)}{\gamma+1}\right\}\right)
$$

sufficiently small such that $f$ is lower semicontinuous on $B_{\varepsilon}((x, y))$ and $B_{\varepsilon}(x) \cap S(f)=$ $\emptyset$. Applying the fuzzy sum rule (Lemma 1(i)), we find points $(z, w) \in X \times Y$ and $\left(x^{*}, y^{*}\right) \in \partial f(z, w)$ such that $d((z, w),(x, y))<\varepsilon, f(z, w)<f(x, y)+\varepsilon$, and $\left\|\left(x^{*}, y^{*}\right)\right\|_{\rho^{\prime}}<$ $\gamma^{\prime}+\varepsilon$. It follows that $d(z, \bar{x})<\rho, 0<f(z, w)<\rho, f(z, w) / d(z, \bar{x})<\gamma,\left\|x^{*}\right\|<\gamma$, and $\left\|y^{*}\right\|<\rho^{\prime} \gamma \leq \rho$. Hence, $|\partial f|_{\rho}(z, w)<\gamma$ and consequently $\overline{|\partial f|}{ }^{+}(\bar{x}, \bar{y}) \leq \gamma$. The claimed inequality follows after letting $\gamma \rightarrow \overline{|\nabla f|}{ }^{+}(\bar{x}, \bar{y})$.

The validity of the equalities under each of the conditions (b)-(d) follows from the proof of [31, Theorem 3.3(iii)] if one replaces the fuzzy sum rule for Fréchet subdifferentials in Asplund spaces there with the exact convex sum rule (Lemma 1(iii)) and differentiable sum rule (Lemma 1(ii)) valid in arbitrary normed spaces.

(vii) Let $\rho>0,(x, y)$ belong to a convex neighbourhood of $(\bar{x}, \bar{y})$ on which $f$ is convex and $0<f(x, y)<\infty$. Take any $(u, v) \in X \times Y$ with $f(u, v)<f(x, y)$. Then,

$$
\frac{f(x, y)-f_{+}(u, v)}{d_{\rho}((x, y),(u, v))} \leq \lim _{t \downarrow 0} \frac{f(x, y)-f((1-t)(x, y)+t(u, v))}{d_{\rho}((1-t)(x, y)+t(u, v),(x, y))} \leq|\nabla f|_{\rho}(x, y),
$$

and consequently, $|\nabla f|_{\rho}^{\diamond}(x, y) \leq|\nabla f|_{\rho}(x, y)$. Together with (i), this proves (a). Equality $\overline{|\nabla f|}^{\diamond}(\bar{x}, \bar{y})=\overline{|\nabla f|}^{>}(\bar{x}, \bar{y})$ follows from (a) and definitions (11) and (13). The other equalities in (b) follow from (ii) and (vi)(b).

Remark 3 One of the main tools in the proof of inequalities

$$
\overline{|\nabla f|}^{>}(\bar{x}, \bar{y}) \geq \overline{|\partial f|}^{>}(\bar{x}, \bar{y}), \quad \overline{|\nabla f|}^{>+}{ }_{(\bar{x}, \bar{y})} \geq \overline{|\partial f|}^{>+}{ }_{(\bar{x}, \bar{y})}
$$

in item (a) of part (vi) of the above proposition, which is crucial for the subdifferential sufficient error bound criteria in Corollaries 1 and 2 below, is the fuzzy sum rule (Lemma 1) for Fréchet subdifferentials in Asplund spaces. There are two ways of extending this inequality to general Banach spaces.

1) Restricting the class of functions to those possessing a kind of sum rule for Fréchet subdifferentials as in items (b)-(d) of part (vi) of the above proposition.

2) Replacing Fréchet subdifferentials with some other (possibly abstract) subdifferentials on the given space satisfying a certain set of natural properties including a kind of (fuzzy or exact) sum rule (trustworthy subdifferentials $[23,24]$ ); cf. [2, Proposition 1.13], [3, Proposition 2.3], [4, Proposition 4.1], [13, Proposition 6]. For instance, one can use for that purpose Ioffe approximate or Clarke-Rockafellar subdifferentials. Note that the opposite inequalities in part (v) are specific for Fréchet subdifferentials and fail in general for other types of subdifferentials. 
The uniform strict outer slope (11) provides the necessary and sufficient characterization of error bounds [31, Theorem 4.1].

Theorem 1 (i) $\operatorname{Er} f(\bar{x}, \bar{y}) \leq \overline{\mid \nabla f}^{\diamond}(\bar{x}, \bar{y})$;

(ii) if $X$ and $Y$ are complete and $f_{+}$is lower semicontinuous near $(\bar{x}, \bar{y})$, then $\operatorname{Er} f(\bar{x}, \bar{y})=$ $\overline{|\nabla f|^{\diamond}}(\bar{x}, \bar{y})$.

It follows from Theorem 1 that inequality $\overline{|\nabla f|^{\diamond}}(\bar{x}, \bar{y})>0$ is crucial for determining the error bound property of $f$ at $(\bar{x}, \bar{y})$.

Remark 4 The nonlocal $\rho$-slope (10) depends on the choice of $\rho$-metric on the product space. If instead of the maximum-type metric $d_{\rho}$, defined by (8), one employs in (10) the sum-type metric $d_{\rho}^{1}$, defined by (9), it will produce a different number. We say that a $\rho$-metric $d_{\rho}^{\prime}$ on $X \times Y$ is admissible if $d_{\rho} \leq d_{\rho}^{\prime} \leq d_{\rho}^{1}$. Thanks to [31, Proposition 4.2], Theorem 1 is invariant on the choice of an admissible $\rho$-metric.

Thanks to Theorem 1 and Proposition 2, one can formulate several quantitative and qualitative criteria of the error bound property in terms of various slopes.

Corollary 1 (Quantitative criteria) Let $\gamma>0$. Consider the following conditions:

(a) $f$ has an error bound with respect to $x$ at $(\bar{x}, \bar{y})$ with some $\tau>0$;

(b) $\overline{|\nabla f|^{\diamond}}(\bar{x}, \bar{y})>\gamma$,

i.e., for some $\rho>0$ and any $(x, y) \in X \times Y$ with $d(x, \bar{x})<\rho$ and $0<f(x, y)<\rho$, it holds $|\nabla f|_{\rho}^{\diamond}(x, y)>\gamma$, and consequently there is a $(u, v) \in X \times Y$ such that

$$
f(x, y)-f_{+}(u, v)>\gamma d_{\rho}((u, v),(x, y)) ;
$$

(c) $\liminf _{x \rightarrow \bar{x}, f(x, y) \downarrow 0} \frac{f(x, y)}{d(x, \bar{x})}>\gamma$;

(d) $\overline{|\nabla f|}>(\bar{x}, \bar{y})>\gamma$,

i.e., for some $\rho>0$ and any $(x, y) \in X \times Y$ with $d(x, \bar{x})<\rho$ and $0<f(x, y)<\rho$, it holds $|\nabla f|_{\rho}(x, y)>\gamma$ and consequently, for any $\varepsilon>0$, there is $a(u, v) \in B_{\varepsilon}(x, y)$ such that

$$
f(x, y)-f(u, v)>\gamma d_{\rho}((u, v),(x, y))
$$

(e) $\left.\overline{|\nabla f|}{ }^{+}{ }_{(\bar{x}}, \bar{y}\right)>\gamma$

i.e., for some $\rho>0$ and any $(x, y) \in X \times Y$ with $d(x, \bar{x})<\rho, 0<f(x, y)<\rho$, and $f(x, y) / d(x, \bar{x}) \leq \gamma$, it holds $|\nabla f|_{\rho}(x, y)>\gamma$ and consequently, for any $\varepsilon>0$, there is a $(u, v) \in B_{\varepsilon}(x, y)$ such that (19) holds true;

(f) $X$ and $Y$ are normed spaces and $\overline{|\partial f|}(\bar{x}, \bar{y})>\gamma$, i.e., for some $\rho>0$ and any $(x, y) \in X \times Y$ with $\|x-\bar{x}\|<\rho$ and $0<f(x, y)<\rho$, it holds $|\partial f|_{\rho}(x, y)>\gamma$ and consequently $\left\|x^{*}\right\|>\gamma$ for all $\left(x^{*}, y^{*}\right) \in \partial f(x, y)$ with $\left\|y^{*}\right\|<\rho$;

(g) $X$ and $Y$ are normed spaces and $\overline{|\partial f|}^{>+}(\bar{x}, \bar{y})>\gamma$,

i.e., for some $\rho>0$ and any $(x, y) \in X \times Y$ with $\|x-\bar{x}\|<\rho, 0<f(x, y)<\rho$, and $f(x, y) /\|x-\bar{x}\| \leq \gamma$, it holds $|\partial f|_{\rho}(x, y)>\gamma$ and consequently $\left\|x^{*}\right\|>\gamma$ for all $\left(x^{*}, y^{*}\right) \in \partial f(x, y)$ with $\left\|y^{*}\right\|<\rho$.

The following implications hold true:

(i) (c) $\Rightarrow$ (e), (d) $\Rightarrow$ (e), (e) $\Rightarrow$ (b), (f) $\Rightarrow($ g) ; 
(ii) if $\gamma<\tau$, then (a) $\Rightarrow$ (b);

(iii) if $\tau \leq \gamma, X$ and $Y$ are complete, and $f_{+}$is lower semicontinuous near $(\bar{x}, \bar{y})$, then (b) $\Rightarrow$ (a).

Suppose $X$ and $Y$ are normed spaces.

(iv) (d) $\Rightarrow$ (f) and (e) $\Rightarrow$ (g);

(v) (d) $\Leftrightarrow(\mathrm{f}) \Rightarrow(\mathrm{e}) \Leftrightarrow$ (g) provided that one of the conditions (a)-(d) in part (vi) of Proposition 2 is satisfied;

(vi) if $f$ is convex near $(\bar{x}, \bar{y})$, then $(\mathrm{b}) \Leftrightarrow(\mathrm{d}) \Leftrightarrow(\mathrm{e}) \Leftrightarrow(\mathrm{f}) \Leftrightarrow(\mathrm{g})$.

The conclusions of Corollary 1 are illustrated in Fig. 1.

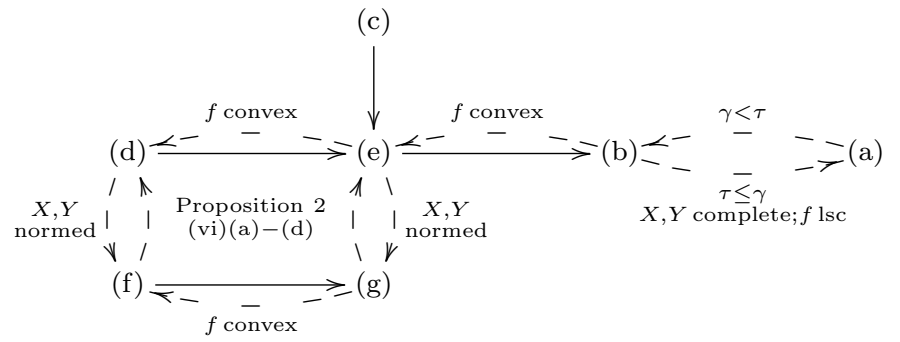

Fig. 1 Corollary 1

Corollary 2 (Qualitative criteria) Suppose $X$ and $Y$ are complete metric spaces and $f_{+}$is lower semicontinuous near $(\bar{x}, \bar{y})$. Then, $f$ has an error bound with respect to $x$ at $(\bar{x}, \bar{y})$ provided that one of the following conditions holds true:

(a) $\overline{|\nabla f|^{\diamond}}(\bar{x}, \bar{y})>0$;

(b) $\liminf _{x \rightarrow \bar{x}, f(x, y) \downarrow 0} \frac{f(x, y)}{d(x, \bar{x})}>0$;

(c) $\overline{|\nabla f|}>(\bar{x}, \bar{y})>0$;

(d) $\mid \overline{|\nabla f|}>+(\bar{x}, \bar{y})>0$, or equivalently,

$$
\lim _{\rho \downarrow 0} \inf _{d(x, \bar{x})<\rho, 0<\frac{f(x, y)}{d(x, \bar{x})}<\rho}|\nabla f|_{\rho}(x, y)>0 .
$$

If $X$ and $Y$ are Banach spaces and one of the conditions (a)-(d) in part (vi) of Proposition 2 is satisfied, then the following conditions are also sufficient:

(e) $\overline{|\partial f|}>(\bar{x}, \bar{y})>0$;

(f) $\overline{|\partial f|}>+(\bar{x}, \bar{y})>0$, or equivalently,

$$
\lim _{\rho \downarrow 0} \inf _{\|x-\bar{x}\|<\rho, 0<\frac{f(x, y)}{\|x-\bar{x}\|}<\rho}|\partial f|_{\rho}(x, y)>0 .
$$

Moreover,

(i) condition (a) is also necessary for the local error bound property of $f$ at $(\bar{x}, \bar{y})$;

(ii) (b) $\Rightarrow$ (d), (c) $\Rightarrow$ (d), (d) $\Rightarrow$ (a), (e) $\Rightarrow$ (f). 
Suppose $X$ and $Y$ are Banach spaces and one of the conditions (a)-(d) in part (vi) of Proposition 2 is satisfied.

(iii) (c) $\Leftrightarrow$ (e) and (d) $\Leftrightarrow$ (f);

(iv) if $f$ is convex near $(\bar{x}, \bar{y})$, then (a) $\Leftrightarrow(\mathrm{c}) \Leftrightarrow(\mathrm{d}) \Leftrightarrow(\mathrm{e}) \Leftrightarrow(\mathrm{f})$.

The conclusions of Corollary 2 are illustrated in Fig. 2.

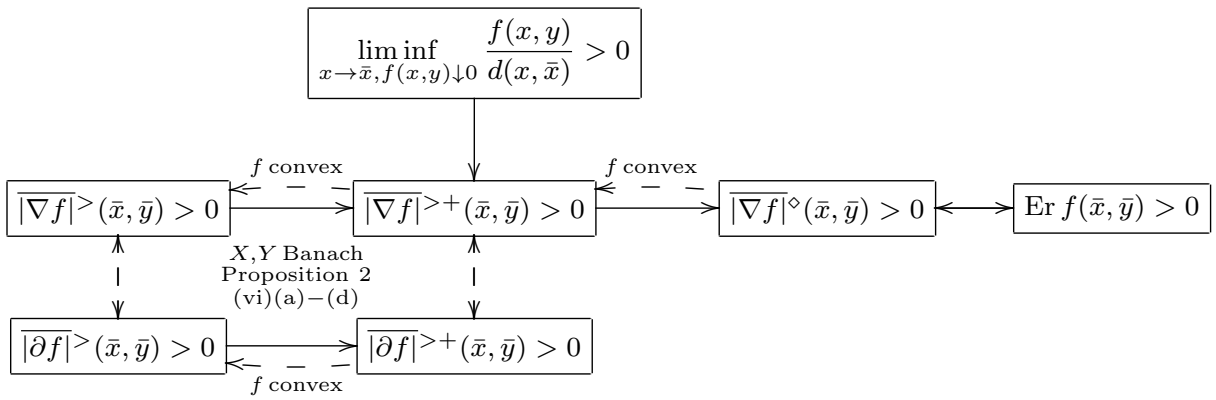

Fig. 2 Corollary 2

\section{Hölder metric subregularity}

From now on, $F: X \rightrightarrows Y$ is a set-valued mapping between metric spaces and $(\bar{x}, \bar{y}) \in$ gph $F$. We are targeting the Hölder metric subregularity property, the main tool being the error bound criteria discussed in the previous section.

\subsection{Definition}

Let a real number $q \in(0,1]$ be given.

A set-valued mapping $F: X \rightrightarrows Y$ between metric spaces is called Hölder metrically subregular of order $q$ at $(\bar{x}, \bar{y}) \in \operatorname{gph} F$ with constant $\tau>0$ if there exists a neighbourhood $U$ of $\bar{x}$ such that

$$
\tau d\left(x, F^{-1}(\bar{y})\right) \leq(d(\bar{y}, F(x)))^{q} \quad \text { for all } x \in U,
$$

or equivalently,

$$
\tau d\left(x, F^{-1}(\bar{y})\right) \leq(d(y, \bar{y}))^{q} \text { for all } x \in U, y \in F(x) .
$$

The Hölder metric subregularity property can be characterized using the following (possibly infinite) constant:

$$
{ }^{s} r_{q}[F](\bar{x}, \bar{y}):=\liminf _{\substack{x \rightarrow \bar{x} \\ x \notin F^{-1}(\bar{y})}} \frac{(d(\bar{y}, F(x)))^{q}}{d\left(x, F^{-1}(\bar{y})\right)},
$$


which coincides with the supremum of all positive $\tau$ such that (20) holds for some $U$ and, when positive, provides a quantitative characterization of this property.

Property (20) is exactly the error bound property (6) for the function $f$ defined by

$$
f(x, y):= \begin{cases}(d(y, \bar{y}))^{q} & \text { if }(x, y) \in \operatorname{gph} F \\ +\infty & \text { otherwise }\end{cases}
$$

while constant $(21)$ coincides (7). Indeed, $f(\bar{x}, \bar{y})=0$, conditions (P1) and (P2) are trivially satisfied, and $f(x, y)=0$ if and only if $y=\bar{y}$ and $x \in F^{-1}(\bar{y})$. Hence, $S(f)=F^{-1}(\bar{y})$. Observe also that condition $f(x, y) \downarrow 0$ is equivalent to $y \rightarrow \bar{y}$ with $(x, y) \in \operatorname{gph} F$ and $y \neq \bar{y}$.

\subsection{Primal space slopes}

The nonlocal slopes (10) and (11) of $f$ in the current setting take the following form:

$$
\begin{gathered}
|\nabla F|_{q, \rho}^{\diamond}(x, y):=\sup _{\substack{(u, v) \neq(x, y) \\
(u, v) \in \operatorname{gph} F}} \frac{\left[(d(y, \bar{y}))^{q}-(d(v, \bar{y}))^{q}\right]+}{d \rho((u, v),(x, y))}, \\
\left.\left|\overline{|\nabla F|_{q}^{\diamond}}(\bar{x}, \bar{y}):=\lim _{\rho \downarrow 0} \inf _{\substack{d(x, \bar{x})<\rho, d(y, \bar{y})<\rho \\
(x, y) \in \operatorname{gph} F, x \notin F^{-1}(\bar{y})}}\right| \nabla F\right|_{q, \rho} ^{\diamond}(x, y) .
\end{gathered}
$$

We will call the above constants, respectively, the nonlocal $(q, \rho)$-slope of $F$ at $(x, y) \in$ gph $F$ and the uniform strict $q$-slope of $F$ at $(\bar{x}, \bar{y})$.

As the main primal space local tool, in this section we are going to use the $\rho$-slope of $F$ at $(x, y)$ :

$$
|\nabla F|_{\rho}(x, y):=\limsup _{\substack{(u, v) \rightarrow(x, y),(u, v) \neq(x, y) \\(u, v) \in \operatorname{gph} F}} \frac{[d(y, \bar{y})-d(v, \bar{y})]_{+}}{d_{\rho}((u, v),(x, y))}
$$

defined for all $\rho>0$ and $(x, y) \in \operatorname{gph} F$. Observe that constant (25) does not depend on $q$.

Using some simple calculus, one can formulate the representation for the local $\rho$ slope (12) in the case when $f$ is given by (22).

Proposition 3 Suppose $(x, y) \in \operatorname{gph} F, y \neq \bar{y}, \rho>0$, and $f$ is given by (22). Then,

$$
|\nabla f|_{\rho}(x, y)=q(d(y, \bar{y}))^{q-1}|\nabla F|_{\rho}(x, y) .
$$

Proof By (12), (22), and (25),

$$
\begin{aligned}
|\nabla f|_{\rho}(x, y) & =\limsup _{\substack{(u, v) \rightarrow(x, y),(u, v) \neq(x, y) \\
(u, v) \in \operatorname{gph} F}} \frac{\left[(d(y, \bar{y}))^{q}-(d(v, \bar{y}))^{q}\right]_{+}}{d_{\rho}((u, v),(x, y))} \\
& =\limsup _{\substack{(u, v) \rightarrow(x, y),(u, v) \neq(x, y) \\
(u, v) \in \operatorname{gph} F}} \frac{\left[q(d(y, \bar{y}))^{q-1}(d(y, \bar{y})-d(v, \bar{y}))+o(d(v, y))\right]_{+}}{d_{\rho}((u, v),(x, y))} \\
& =q(d(y, \bar{y}))^{q-1} \limsup _{\substack{(u, v) \rightarrow(x, y),(u, v) \neq(x, y) \\
(u, v) \in \operatorname{gph} F}} \frac{[d(y, \bar{y})-d(v, \bar{y})]_{+}}{d_{\rho}((u, v),(x, y))} \\
& =q(d(y, \bar{y}))^{q-1}|\nabla F|_{\rho}(x, y) .
\end{aligned}
$$


In the above formula, $o(\cdot)$ is a function from $\mathbb{R}_{+}$to $\mathbb{R}_{+}$with the property $o(t) / t \rightarrow 0$ as $t \downarrow 0$.

The strict q-slope and the modified strict $q$-slope of $F$ at $(\bar{x}, \bar{y})$ are defined as follows:

$$
\begin{aligned}
& \overline{\mid \nabla F}_{q}(\bar{x}, \bar{y}):=q \lim _{\rho \downarrow 0} \inf _{\substack{d(x, \bar{x})<\rho, d(y, \bar{y})<\rho \\
(x, y) \in \operatorname{gph} F, x \notin F^{-1}(\bar{y})}}(d(y, \bar{y}))^{q-1}|\nabla F|_{\rho}(x, y), \\
& \overline{|\nabla F|}_{q}^{+}(\bar{x}, \bar{y}):=\lim _{\rho \downarrow 0} \inf _{\substack{d(x, \bar{x})<\rho, d(y, \bar{y})<\rho \\
(x, y) \in \operatorname{gph} F, x \notin F^{-1}(\bar{y})}} \max \left\{q(d(y, \bar{y}))^{q-1}|\nabla F|_{\rho}(x, y), \frac{(d(y, \bar{y}))^{q}}{d(x, \bar{x})}\right\} .
\end{aligned}
$$

In view of Proposition 3, these constants coincide, respectively, with the corresponding strict outer slopes (13) and (14) of $f$.

\subsection{Subdifferential slopes}

In this subsection, $X$ and $Y$ are normed spaces. We define, respectively, the subdifferential $\rho$-slope and the approximate subdifferential $\rho$-slope $(\rho>0)$ of $F$ at $(x, y) \in \operatorname{gph} F$ with $y \neq \bar{y}$ as

$$
\begin{gathered}
|\partial F|_{\rho}(x, y):=\operatorname{linf}_{x^{*} \in D^{*} F(x, y)\left(J(y-\bar{y})+\rho \mathbb{B}^{*}\right)}\left\|x^{*}\right\|, \\
|\partial F|_{\rho}^{a}(x, y):=\liminf _{v \rightarrow y-\bar{y}} \inf _{x^{*} \in D^{*} F(x, y)\left(J(v)+\rho \mathbb{B}^{*}\right)}\left\|x^{*}\right\|,
\end{gathered}
$$

where $J$ is the duality mapping defined by (5). These constants do not depend on $q$.

In the rest of the section, when $Y$ is a normed space, we use the notation

$$
\xi_{q}(y):=\|y-\bar{y}\|^{1-q} / q .
$$

The next proposition gives representations for the subdifferential $\rho$-slope (15) in the case when $f$ is given by $(22)$.

Proposition 4 Suppose $(x, y) \in \operatorname{gph} F, y \neq \bar{y}, \rho>0$, and $f$ is given by (22).

(i) If $X$ and $Y$ are Asplund and gph $F$ is locally closed near $(x, y)$, then

$$
|\partial f|_{\rho}(x, y) \geq q\|y-\bar{y}\|^{q-1} \liminf _{\substack{\left(x^{\prime}, y^{\prime}\right) \rightarrow(x, y), y^{\prime \prime} \rightarrow y \\\left(x^{\prime}, y^{\prime}\right) \in \operatorname{gph} F}} \inf ^{* \in D^{*} F\left(x^{\prime}, y^{\prime}\right)\left(J\left(y^{\prime \prime}-\bar{y}\right)+\xi_{q}\left(y^{\prime \prime}\right) \rho \mathbb{B}^{*}\right)}\left\|x^{*}\right\| .
$$

(ii) If either $F$ is convex near $(x, y)$ and $q=1$ or $Y$ is Fréchet smooth, then

$$
|\partial f|_{\rho}(x, y)=q\|y-\bar{y}\|^{q-1}|\partial F|_{\xi_{q}(y) \rho}(x, y) .
$$

Proof (i) Suppose that $X$ and $Y$ are normed spaces and observe that

$$
f(u, v)=g(v)+i_{\operatorname{gph} F}(u, v), \quad(u, v) \in X \times Y,
$$

where $g(v)=\|v-\bar{y}\|^{q}$ and $i_{\mathrm{gph} F}$ is the indicator function of $\operatorname{gph} F: i_{\operatorname{gph} F}(u, v)=0$ if $(u, v) \in \operatorname{gph} F$ and $i_{\operatorname{gph} F}(u, v)=\infty$ otherwise. In its turn, function $g$ is a composition of two functions: $v \mapsto\|v-\bar{y}\|$ on $Y$ and $t \mapsto t^{q}$ on $\mathbb{R}_{+}$. The latter function is Fréchet 
differentiable on $(0, \infty)$. It follows from the composition rule for Fréchet subdifferentials (cf., e.g., [30, Corollary 1.14.1]) that, for any $v \neq \bar{y}$,

$$
\partial g(v)=q\|v-\bar{y}\|^{q-1} J(v-\bar{y})
$$

If $X$ and $Y$ are Asplund, then the fuzzy sum rule (Lemma 1) is applicable to function $f$ : for any $\varepsilon>0$,

$$
\partial f(x, y) \subset \bigcup_{\substack{\left\|\left(x^{\prime}, y^{\prime}\right)-(x, y)\right\|<\varepsilon,\left(x^{\prime}, y^{\prime}\right) \in \operatorname{gph} F \\\left(x^{*}, y^{*}\right) \in N_{\text {gph } F}\left(x^{\prime}, y^{\prime}\right) \\\left\|y^{\prime \prime}-y\right\|<\varepsilon, v^{*} \in \partial g\left(y^{\prime \prime}\right)}}\left\{x^{*}, y^{*}+v^{*}\right\}+\varepsilon \mathbb{B}_{X^{*} \times Y^{*}}
$$

By definition (15),

$$
\begin{aligned}
& |\partial f|_{\rho}(x, y) \geq \lim _{\varepsilon \downarrow 0}\left(\inf _{\left\|\left(x^{\prime}, y^{\prime}\right)-(x, y)\right\|<\varepsilon,\left(x^{\prime}, y^{\prime}\right) \in \operatorname{gph} F}\left\|x^{*}\right\|-\varepsilon\right) \\
& \begin{array}{l}
\left(x^{*}, y^{*}\right) \in N_{\mathrm{gph} F}\left(x^{\prime}, y^{\prime}\right) \\
\left\|y^{\prime \prime}-y\right\|<\varepsilon, v^{*} \in \partial g\left(y^{\prime \prime}\right)
\end{array} \\
& \left\|y^{*}+v^{*}\right\|<\rho \\
& =\lim _{\varepsilon \downarrow 0\left\|\left(x^{\prime}, y^{\prime}\right)-(x, y)\right\|<\varepsilon,\left(x^{\prime}, y^{\prime}\right) \in \operatorname{gph} F}\left\|x^{*}\right\| \\
& \begin{array}{c}
x^{*} \in D^{*} F\left(x^{\prime}, y^{\prime}\right)\left(y^{*}\right) \\
\left\|y^{\prime \prime}-y\right\|<\varepsilon, v^{*} \in \partial g\left(y^{\prime \prime}\right)
\end{array} \\
& \left\|y^{\prime \prime}-y\right\|<\varepsilon, v^{*} \in \partial g\left(y^{\prime \prime}\right) \\
& =\lim _{\varepsilon \downarrow 0\left\|\left(x^{\prime}, y^{\prime}\right)-(x, y)\right\|<\varepsilon,\left(x^{\prime}, y^{\prime}\right) \in \operatorname{gph} F}\left\|x^{*}\right\| \\
& x^{*} \in D^{*} F\left(x^{\prime}, y^{\prime}\right)\left(\partial g\left(y^{\prime \prime}\right)+\rho \mathbb{B}^{*}\right) \\
& =\lim _{\varepsilon \downarrow 0 \|} \inf _{\substack{\left(x^{\prime}, y^{\prime}\right)-(x, y)\left\|<\varepsilon x^{*} \in D^{*} F\left(x^{\prime}, y^{\prime}\right)\left(\partial g\left(y^{\prime \prime}\right)+\rho \mathbb{B}^{*}\right) \\
\left(x^{\prime}, y^{\prime}\right) \in \operatorname{gph} F\\
\right\| y^{\prime \prime}-y \|<\varepsilon}}\left\|x^{*}\right\| \\
& =\liminf _{\substack{\left(x^{\prime}, y^{\prime}\right) \rightarrow(x, y), y^{\prime \prime} \rightarrow y \\
\left(x^{\prime}, y^{\prime}\right) \in \operatorname{gph} F}} \inf _{x^{*} \in D^{*} F\left(x^{\prime}, y^{\prime}\right)\left(\partial g\left(y^{\prime \prime}\right)+\rho \mathbb{B}^{*}\right)}\left\|x^{*}\right\| \\
& =\liminf _{\substack{\left(x^{\prime}, y^{\prime}\right) \rightarrow(x, y), y^{\prime \prime} \rightarrow y \\
\left(x^{\prime}, y^{\prime}\right) \in \operatorname{gph} F}} \inf _{x^{*} \in D^{*} F\left(x^{\prime}, y^{\prime}\right)\left(q\left\|y^{\prime \prime}-\bar{y}\right\|^{q-1} J\left(y^{\prime \prime}-\bar{y}\right)+\rho \mathbb{B}^{*}\right)}\left\|x^{*}\right\| \\
& =\liminf _{\substack{\left(x^{\prime}, y^{\prime}\right) \rightarrow(x, y), y^{\prime \prime} \rightarrow y \\
\left(x^{\prime}, y^{\prime}\right) \in \operatorname{gph} F}} \inf _{x^{*} \in D^{*} F\left(x^{\prime}, y^{\prime}\right)\left(J\left(y^{\prime \prime}-\bar{y}\right)+\xi_{q}\left(y^{\prime \prime}\right) \rho \mathbb{B}^{*}\right)} q\left\|y^{\prime \prime}-\bar{y}\right\|^{q-1}\left\|x^{*}\right\| \\
& =q\|y-\bar{y}\|^{q-1} \liminf _{\substack{\left(x^{\prime}, y^{\prime}\right) \rightarrow(x, y), y^{\prime \prime} \rightarrow y \\
\left(x^{\prime}, y^{\prime}\right) \in \operatorname{gph} F}} \inf _{x^{*} \in D^{*} F\left(x^{\prime}, y^{\prime}\right)\left(J\left(y^{\prime \prime}-\bar{y}\right)+\xi_{q}\left(y^{\prime \prime}\right) \rho \mathbb{B}^{*}\right)}\left\|x^{*}\right\| .
\end{aligned}
$$

(ii) The proof is similar to that of (i). Instead of the fuzzy sum rule, one can use either the differentiable sum rule (part (ii) of Lemma 1) when $Y$ is Fréchet smooth, or the convex sum rule (part (iii) of Lemma 1 ) when $F$ is convex and $q=1$ to write down the representation:

$$
\partial f(x, y)=\bigcup_{\left(x^{*}, y^{*}\right) \in N_{\operatorname{gph} F}(x, y)}\left\{x^{*}, q\|y-\bar{y}\|^{q-1} J(y-\bar{y})+y^{*}\right\},
$$


By definition (15),

$$
\begin{aligned}
|\partial f|_{\rho}(x, y) & \inf _{\substack{\left(x^{*}, y^{*}\right) \in N_{\operatorname{gph} F}(x, y) \\
v^{*} \in J(y-\bar{y}),\left\|y^{*}+q\right\| y-\bar{y}\left\|^{q-1} v^{*}\right\|<\rho}}\left\|x^{*}\right\| \\
& =q\|y-\bar{y}\|^{q-1} \inf _{\substack{\left(x^{*}, y^{*}\right) \in N_{\text {gph } F}(x, y) \\
v^{*} \in J(y-\bar{y}),\left\|y^{*}+v^{*}\right\|<\xi_{q}(y) \rho}}\left\|x^{*}\right\| \\
& =q\|y-\bar{y}\|^{q-1} \inf _{x^{*} \in D^{*} F(x, y)\left(J(y-\bar{y})+\xi_{q}(y) \rho \mathbb{B}^{*}\right)}\left\|x^{*}\right\| \\
& =q\|y-\bar{y}\|^{q-1}|\partial F|_{\xi_{q}(y) \rho}(x, y) .
\end{aligned}
$$

3.4 Strict subdifferential slopes

Using subdifferential $\rho$-slopes (28) and (29), we define now the strict subdifferential $q$-slope and the approximate strict subdifferential q-slope of $F$ at $(\bar{x}, \bar{y})$ :

$$
\begin{aligned}
& \overline{|\partial F|_{q}}(\bar{x}, \bar{y}):=q \lim _{\rho \downarrow 0} \inf _{\substack{\|x-\bar{x}\|<\rho,\|y-\bar{y}\|<\rho \\
(x, y) \in \operatorname{gph} F, x \notin F^{-1}(\bar{y})}}\|y-\bar{y}\|^{q-1}|\partial F|_{\xi_{q}(y) \rho}(x, y), \\
& \overline{|\partial F|}_{q}^{a}(\bar{x}, \bar{y}):=q \lim _{\rho \downarrow 0} \inf _{\substack{\|x-\bar{x}\|<\rho,\|y-\bar{y}\|<\rho \\
(x, y) \in \operatorname{gph} F, x \notin F^{-1}(\bar{y})}}\|y-\bar{y}\|^{q-1}|\partial F|_{\xi_{q}(y) \rho}^{a}(x, y)
\end{aligned}
$$

and their modified analogues:

$$
\begin{aligned}
& \overline{|\partial F|}_{q}^{+}(\bar{x}, \bar{y}):= \\
& \lim _{\rho \downarrow 0} \inf _{\substack{\|x-\bar{x}\|<\rho,\|y-\bar{y}\|<\rho \\
(x, y) \in \operatorname{gph} F, x \notin F^{-1}(\bar{y})}} \max \left\{q\|y-\bar{y}\|^{q-1}|\partial F|_{\xi_{q}(y) \rho}(x, y), \frac{\|y-\bar{y}\|^{q}}{\|x-\bar{x}\|}\right\}, \\
& \overline{|\partial F|}_{q}^{a+}(\bar{x}, \bar{y}):= \\
& \lim _{\rho \downarrow 0} \inf _{\substack{\|x-\bar{x}\|<\rho,\|y-\bar{y}\|<\rho \\
(x, y) \in \operatorname{gph} F, x \notin F^{-1}(\bar{y})}} \max \left\{q\|y-\bar{y}\|^{q-1}|\partial F|_{\xi_{q}(y) \rho}^{a}(x, y), \frac{\|y-\bar{y}\|^{q}}{\|x-\bar{x}\|}\right\} .
\end{aligned}
$$

With the help of Proposition 4, we can formulate representations for the strict outer subdifferential slopes (16) and (17) in the case when $f$ is given by (22).

Proposition 5 Let $f$ be given by (22).

(i) If $X$ and $Y$ are Asplund and $\operatorname{gph} F$ is locally closed near $(\bar{x}, \bar{y})$, then

$$
\left.\overline{|\partial f|}^{>}(\bar{x}, \bar{y}) \geq \overline{|\partial F|}_{q}^{a}(\bar{x}, \bar{y}) \quad \text { and } \quad \overline{\mid \partial f}^{>+}{ }_{(\bar{x}}, \bar{y}\right) \geq{\overline{|\partial F|_{q}^{a}}}^{a+}(\bar{x}, \bar{y}) .
$$

(ii) If either $F$ is convex near $(\bar{x}, \bar{y})$ and $q=1$ or $Y$ is Fréchet smooth, then

$$
\overline{|\partial f|}^{>}(\bar{x}, \bar{y})=\overline{|\partial F|}_{q}(\bar{x}, \bar{y}) \quad \text { and } \quad \overline{|\partial f|}^{>+}(\bar{x}, \bar{y})=\overline{\mid \partial F}_{q}^{+}(\bar{x}, \bar{y}) \text {. }
$$


Proof (i) By (16) and Proposition 4(i),

$$
\begin{aligned}
& \overline{|\partial f|}^{>}(\bar{x}, \bar{y}) \geq \lim _{\rho \downarrow 0} \inf _{\substack{\|(x, y)-(\bar{x}, \bar{y})\|<\rho \\
(x, y) \in \operatorname{gph} F, x \notin F^{-1}(\bar{y})}} \\
& q\|y-\bar{y}\|^{q-1} \lim _{\varepsilon \downarrow 0} \inf _{\substack{\left\|\left(x^{\prime}, y^{\prime}\right)-(x, y)\right\|<\varepsilon,\left\|y^{\prime \prime}-y\right\|<\varepsilon \\
x^{*} \in D^{*} F\left(x^{\prime}, y^{\prime}\right)\left(J\left(y^{\prime \prime}-\bar{y}\right)+\xi_{q}\left(y^{\prime \prime}\right) \rho \mathbb{B}^{*}\right) \\
\left(x^{\prime}, y^{\prime}\right) \in \operatorname{gph} F}}\left\|x^{*}\right\| .
\end{aligned}
$$

For fixed $\rho \in(0,1)$ and $(x, y)$ with $x \notin F^{-1}(\bar{y})$ and a sufficiently small $\varepsilon>0$, it holds $B_{\varepsilon}(x) \cap F^{-1}(\bar{y})=\emptyset,\|(x, y)-(\bar{x}, \bar{y})\|+\varepsilon<\rho$, and $\|y-\bar{y}\|^{q-1} \geq(1-\rho)\left\|y^{\prime}-\bar{y}\right\|^{q-1}$ for all $y^{\prime} \in B_{\varepsilon}(y)$. Besides, $\left\|y^{\prime \prime}-y^{\prime}\right\| \leq\left\|y^{\prime \prime}-y\right\|+\left\|y^{\prime}-y\right\|<2 \varepsilon$. Hence,

$$
\begin{gathered}
\overline{|\partial f|}^{>}(\bar{x}, \bar{y}) \geq q \lim _{\rho \downarrow 0} \underset{\substack{\left\|\left(x^{\prime}, y^{\prime}\right)-(\bar{x}, \bar{y})\right\|<\rho \\
\left(x^{\prime}, y^{\prime}\right) \in \operatorname{gph} F, x^{\prime} \notin F^{-1}(\bar{y})}}{(1-\rho)\left\|y^{\prime}-\bar{y}\right\|^{q-1} \lim _{\varepsilon \downarrow 0} \inf _{\substack{x^{*} \in D^{*} F\left(x^{\prime}, y^{\prime}\right)\left(J\left(y^{\prime \prime}-\bar{y}\right)+\xi_{q}\left(y^{\prime \prime}\right) \rho \mathbb{B}^{*}\right) \\
x^{\prime} \|<2 \varepsilon}}\left\|x^{*}\right\|} \\
=q \lim _{\rho \downarrow 0} \underset{\substack{\left\|\left(x^{\prime}, y^{\prime}\right)-(\bar{x}, \bar{y})\right\|<\rho \\
\left(x^{\prime}, y^{\prime}\right) \in \operatorname{gph} F, x^{\prime} \notin F^{-1}(\bar{y})}}{\left\|y^{\prime}-\bar{y}\right\|^{q-1}|\partial F|_{\xi_{q}\left(y^{\prime}\right) \rho}^{a}(x, y)=\overline{|\partial F|}_{q}^{a}(\bar{x}, \bar{y}) .}
\end{gathered}
$$

The proof of the other inequality goes along the same lines.

(ii) follows from Proposition 4(ii) in view of definitions (16), (17), (30), and (32).

The next proposition provides some simplifications in the representations (30)-(33).

Proposition 6 The following assertions hold true:

(i) $\overline{|\partial F|}_{q}(\bar{x}, \bar{y}) \geq q \lim _{\rho \downarrow 0} \inf _{\|x-\bar{x}\|<\rho,\|y-\bar{y}\|<\rho}\|y-\bar{y}\|^{q-1}|\partial F|_{\rho}(x, y)$;

(ii) $\overline{|\partial F|}_{q}^{a}(\bar{x}, \bar{y}) \geq q \lim _{\rho \downarrow 0} \inf _{\|x-\bar{x}\|<\rho,\|y-\bar{y}\|<\rho}\|y-\bar{y}\|^{q-1}|\partial F|_{\rho}^{a}(x, y)$; $(x, y) \in \operatorname{gph} F, x \notin F^{-1}(\bar{y})$

(iii) $\overline{\mid \partial F}_{q}^{+}(\bar{x}, \bar{y}) \geq \lim _{\rho \downarrow 0} \inf _{\substack{\|x-\bar{x}\|<\rho,\|y-\bar{y}\|<\rho \\(x, y) \in \operatorname{gph} F, x \notin F^{-1}(\bar{y})}} \max \left\{q\|y-\bar{y}\|^{q-1}|\partial F|_{\rho}(x, y), \frac{\|y-\bar{y}\|^{q}}{\|x-\bar{x}\|}\right\}$; (iv) ${\overline{|\partial F|_{q}^{a}}}_{q}^{a+}(\bar{x}, \bar{y}) \geq \lim _{\rho \downarrow 0} \inf _{\substack{\|x-\bar{x}\|<\rho,\|y-\bar{y}\|<\rho \\(x, y) \in \operatorname{gph} F, x \notin F^{-1}(\bar{y})}} \max \left\{q\|y-\bar{y}\|^{q-1}|\partial F|_{\rho}^{a}(x, y), \frac{\|y-\bar{y}\|^{q}}{\|x-\bar{x}\|}\right\}$.

If $q=1$, the above relations hold as equalities.

Proof We consider the first inequality. The others can be treated in the same way. If $\overline{\mid \partial F}_{q}(\bar{x}, \bar{y})=\infty$, the inequality holds trivially. Let $\overline{\mid \partial F}_{q}(\bar{x}, \bar{y})<\gamma<\infty$. Fix an arbitrary $\rho>0$ and choose an $\alpha>0$ and a $\rho^{\prime} \in(0, \rho)$ such that $\alpha\left(\rho^{\prime}\right)^{1-q}<q$ and $\rho^{\prime}<\alpha \rho$. By (30), there exists an $(x, y) \in \operatorname{gph} F$ with $\|x-\bar{x}\|<\rho^{\prime},\|y-\bar{y}\|<\rho^{\prime}$ and $x \notin F^{-1}(\bar{y})$; a $y^{*} \in Y^{*}$, an $x^{*} \in D^{*} F(x, y)\left(y^{*}\right)$, and a $v^{*} \in J(y-\bar{y})$ such that $\left\|v^{*}-y^{*}\right\| \leq\|y-\bar{y}\|^{1-q} \rho^{\prime} / q$ and $q\|y-\bar{y}\|^{q-1}\left\|x^{*}\right\|<\gamma$. Hence, $\|x-\bar{x}\|<\rho,\|y-\bar{y}\|<\rho$, and $\left\|v^{*}-y^{*}\right\| \leq \alpha^{-1} \rho^{\prime}<\rho$, and consequently the right-hand side of (i) is less than $\gamma$. The conclusion follows since $\gamma$ was chosen arbitrarily.

If $q=1$, then the right-hand sides of (i) and (30) coincide. 
The next statement summarizes the relationships between the $q$-slopes. It is a consequence of the definitions and Propositions 3 and 5.

\section{Proposition 7 (Relationships between slopes)}

(i) $|\nabla F|_{q, \rho}^{\diamond}(x, y) \geq \max \left\{q(d(y, \bar{y}))^{q-1}|\nabla F|_{\rho}(x, y), \frac{(d(y, \bar{y}))^{q}}{d_{\rho}((x, y),(\bar{x}, \bar{y}))}\right\}$ for all $\rho>0$ and $(x, y) \in \operatorname{gph} F$ with $y \neq \bar{y}$;

(ii) $\overline{\mid \nabla F}_{q}^{\diamond}(\bar{x}, \bar{y}) \geq \overline{\mid \nabla F}_{q}^{+}(\bar{x}, \bar{y}) \geq \overline{|\nabla F|}_{q}(\bar{x}, \bar{y})$.

Suppose $X$ and $Y$ are normed spaces.

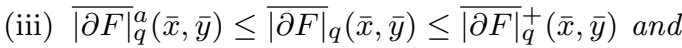
$\overline{\mid \partial F}_{q}^{a}(\bar{x}, \bar{y}) \leq{\overline{|\partial F|_{q}^{a}}}^{+}(\bar{x}, \bar{y}) \leq \overline{\mid \partial F}_{q}^{+}(\bar{x}, \bar{y}) ;$

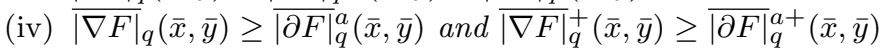
provided that $X$ and $Y$ are Asplund and gph $F$ is locally closed near $(\bar{x}, \bar{y})$;

(v) $\overline{|\nabla F|}_{q}(\bar{x}, \bar{y})=\overline{|\partial F|}_{q}(\bar{x}, \bar{y})$ and $\overline{|\nabla F|_{q}^{+}}(\bar{x}, \bar{y})=\overline{\mid \partial F}_{q}^{+}(\bar{x}, \bar{y})$ provided that $Y$ is Fréchet smooth and one of the following conditions is satisfied: (a) $X$ is Asplund and gph $F$ is locally closed near $(\bar{x}, \bar{y})$;

(b) $F$ is convex near $(\bar{x}, \bar{y})$;

(vi) $\overline{|\nabla F|_{1}^{\diamond}}(\bar{x}, \bar{y})=\overline{\mid \nabla F}_{1}^{+}(\bar{x}, \bar{y})=\overline{|\nabla F|}(\bar{x}, \bar{y})=\overline{\mid \partial F}_{1}^{+}(\bar{x}, \bar{y})=\overline{\mid \partial F}_{1}(\bar{x}, \bar{y})$ provided that $F$ is convex near $(\bar{x}, \bar{y})$.

3.5 Limiting outer $q$-coderivative

In this subsection, $X$ and $Y$ are finite dimensional normed linear spaces.

In finite dimensions, strict subdifferential $q$-slopes (30) and (31) coincide and can be equivalently expressed in terms of the limiting outer $q$-coderivative $\bar{D}_{q}^{*>} F(\bar{x}, \bar{y})$ of $F$ at $(\bar{x}, \bar{y})$ defined by its graph as follows:

$$
\begin{aligned}
\operatorname{gph} \bar{D}_{q}^{*>} F(\bar{x}, \bar{y}):=\left\{\left(y^{*}, x^{*}\right) \in Y^{*} \times X^{*} \mid\right. \\
\exists\left(x_{k}, y_{k}, x_{k}^{*}, y_{k}^{*}, v_{k}^{*}\right) \subset X \times Y \times X^{*} \times Y^{*} \times Y^{*} \text { such that } \\
\left(x_{k}, y_{k}\right) \in \operatorname{gph} F, x_{k} \notin F^{-1}(\bar{y}), \\
\left(y_{k}^{*}, x_{k}^{*}\right) \in \operatorname{gph} D^{*} F\left(x_{k}, y_{k}\right), v_{k}^{*} \in J\left(y_{k}-\bar{y}\right), \\
\left(x_{k}, y_{k}\right) \rightarrow(\bar{x}, \bar{y}), y_{k}^{*}-q\left\|y_{k}-\bar{y}\right\|^{q-1} v_{k}^{*} \rightarrow 0, \\
\left.\left\|y^{*}\right\| x_{k}^{*} \rightarrow x^{*}, \text { if } y^{*} \neq 0, \text { then } \frac{y_{k}^{*}}{\left\|y_{k}^{*}\right\|} \rightarrow \frac{y^{*}}{\left\|y^{*}\right\|}\right\} .
\end{aligned}
$$

This set is a closed cone in $X \times Y$. Hence, the limiting outer $q$-coderivative is a closed positively homogeneous set-valued mapping.

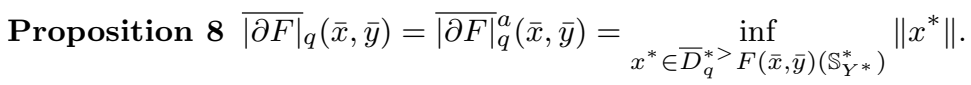

Proof We first prove that

$$
\overline{|\partial F|}_{q}(\bar{x}, \bar{y})=\inf _{x^{*} \in \bar{D}_{q}^{*>} F(\bar{x}, \bar{y})\left(\mathbb{S}_{Y^{*}}^{*}\right)}\left\|x^{*}\right\| .
$$


Let $\left(y^{*}, x^{*}\right) \in \operatorname{gph} \bar{D}_{q}^{*>} F(\bar{x}, \bar{y}),\left\|y^{*}\right\|=1$, and $\rho>0$. Choose an arbitrary sequence $\left(x_{k}, y_{k}, x_{k}^{*}, y_{k}^{*}, v_{k}^{*}, \alpha_{k}\right)$ corresponding to $\left(y^{*}, x^{*}\right)$ in accordance with definition (34). Then, for a sufficiently large $k$, it holds $\left\|x_{k}-\bar{x}\right\|<\rho,\left\|y_{k}-\bar{y}\right\|<\rho,\left(x_{k}, y_{k}\right) \in$ $\operatorname{gph} F, x_{k} \notin F^{-1}(\bar{y}), y_{k}^{*} \in q\left\|y_{k}-\bar{y}\right\|^{q-1} J\left(y_{k}-\bar{y}\right)+\rho \mathbb{B}^{*}, x_{k}^{*} \in D^{*} F\left(x_{k}, y_{k}\right)\left(y_{k}^{*}\right)$, and $\left\|x_{k}^{*}-x^{*}\right\|<\rho$. Hence, by (28) and (30), $\overline{|\partial F|}(\bar{x}, \bar{y}) \leq\left\|x_{k}^{*}\right\|<\left\|x^{*}\right\|+\rho$ and consequently,

$$
\overline{|\partial F|}_{q}(\bar{x}, \bar{y}) \leq \inf _{x^{*} \in \bar{D}_{q}^{*>} F(\bar{x}, \bar{y})\left(\mathbb{S}_{Y^{*}}^{*}\right)}\left\|x^{*}\right\| .
$$

Conversely, by definitions (28) and (30), there exist sequences $\left(x_{k}, y_{k}\right) \rightarrow(\bar{x}, \bar{y})$ with $\left(x_{k}, y_{k}\right) \in \operatorname{gph} F, x_{k} \notin F^{-1}(\bar{y})$ and $\left(x_{k}^{*}, y_{k}^{*}, v_{k}^{*}\right) \in X^{*} \times Y^{*} \times Y^{*}$ with $\left(y_{k}^{*}, x_{k}^{*}\right) \in$ $\operatorname{gph} D^{*} F\left(x_{k}, y_{k}\right), v_{k}^{*} \in J\left(y_{k}-\bar{y}\right)$ such that $y_{k}^{*}-q\left\|y_{k}-\bar{y}\right\|^{q-1} v_{k}^{*} \rightarrow 0$ and $\left\|x_{k}^{*}\right\| \rightarrow$ $\overline{\mid \partial F}_{q}(\bar{x}, \bar{y})$. Passing to subsequences if necessary, we can assume that $x_{k}^{*} \rightarrow x^{*} \in X^{*}$ and either $y_{k}^{*} \neq 0$ for all $k \in \mathbb{N}$, or $y_{k}^{*}=0$ for all $k \in \mathbb{N}$. In the first case, we can assume that $y_{k}^{*} /\left\|y_{k}^{*}\right\| \rightarrow y^{*} \in \mathbb{S}_{Y^{*}}^{*}$, and consequently, by definition (34), $\left(x^{*}, y^{*}\right) \in$ $\operatorname{gph} \bar{D}_{q}^{*>} F(\bar{x}, \bar{y})$. In the second case, $\left(x^{*}, y^{*}\right) \in \operatorname{gph} \bar{D}_{q}^{*>} F(\bar{x}, \bar{y})$ for any $y^{*} \in Y^{*}$. Hence,

$$
\overline{|\partial F|}_{q}(\bar{x}, \bar{y})=\left\|x^{*}\right\| \geq \inf _{x^{*} \in \bar{D}_{q}^{*>} F(\bar{x}, \bar{y})\left(\mathbb{S}_{Y^{*}}^{*}\right)}\left\|x^{*}\right\| .
$$

This together with (36) proves (35).

The remaining equality

$$
\overline{|\partial F|}_{q}^{a}(\bar{x}, \bar{y})=\inf _{x^{*} \in \bar{D}_{q}^{*>} F(\bar{x}, \bar{y})\left(\mathbb{S}_{Y^{*}}^{*}\right)}\left\|x^{*}\right\|
$$

follows from comparing definitions (28) and (29) thanks to the upper semicontinuity of the duality mapping.

Remark 5 The above definition of the limiting outer $q$-coderivative follows the original idea of limiting coderivatives; cf. [36]. In particular, it defines a positively homogeneous set-valued mapping with a not necessarily convex graph. However, there are also several important distinctions. Firstly, similar to the corresponding definition introduced in [26], this is an "outer" object: only sequences $\left(x_{k}, y_{k}\right) \in \operatorname{gph} F$ with $x_{k}$ components lying outside of the set $F^{-1}(\bar{y})$ are taken into account. Secondly, as it is reflected in its name, the limiting outer $q$-coderivative depends on $q$. It is not excluded in the definition that $\left\|v_{k}^{*}\right\| \rightarrow \infty$ and consequently $\left\|y_{k}^{*}\right\| \rightarrow \infty$, and nevertheless the sequence $\left(y_{k}^{*}\right)$ produces a finite element $y^{*} \in Y$.

Remark 6 Analyzing the definition of the limiting outer $q$-coderivative and the proof of Proposition 8 , one can notice that there is no need to care much about the convergence of the sequences in $Y^{*}$. The limiting outer $q$-coderivative in Proposition 8 can be replaced by the corresponding limiting set in $X^{*}$ only:

$$
\begin{aligned}
S_{q}^{*>} F(\bar{x}, \bar{y}):=\left\{x^{*} \in X^{*} \mid \exists\left(x_{k}, y_{k}, x_{k}^{*}, y_{k}^{*}, v_{k}^{*}\right) \subset X \times Y \times X^{*} \times Y^{*} \times Y^{*}\right. \\
\quad \text { such that }\left(x_{k}, y_{k}\right) \in \operatorname{gph} F, x_{k} \notin F^{-1}(\bar{y}),\left(y_{k}^{*}, x_{k}^{*}\right) \in \operatorname{gph} D^{*} F\left(x_{k}, y_{k}\right), \\
\left.\quad v_{k}^{*} \in J\left(y_{k}-\bar{y}\right),\left(x_{k}, y_{k}\right) \rightarrow(\bar{x}, \bar{y}), y_{k}^{*}-q\left\|y_{k}-\bar{y}\right\|^{q-1} v_{k}^{*} \rightarrow 0, x_{k}^{*} \rightarrow x^{*}\right\} .
\end{aligned}
$$

Proposition 8 remains true if $\bar{D}_{q}^{*>} F(\bar{x}, \bar{y})\left(\mathbb{S}_{Y^{*}}^{*}\right)$ there is replaced by $S_{q}^{*>} F(\bar{x}, \bar{y})$. This way one can also relax the assumption that $\operatorname{dim} Y<\infty$. 
Remark 7 One can define also a $q$-coderivative (indirect) counterpart of the modified strict subdifferential $q$-slopes (32) and (33). It is sufficient to add to the list of properties in definition (34) an additional requirement that $\left\|y_{k}-\bar{y}\right\|^{q} /\left\|x_{k}-\bar{x}\right\| \rightarrow 0$ as $k \rightarrow \infty$. The corresponding set can be used for characterizing metric $q$-subregularity. However, the analogues of the equalities in Proposition 8 would not hold for it.

\section{Criteria of Hölder metric subregularity}

In this section, if not specified otherwise, $X$ and $Y$ are metric spaces. The next theorem is a consequence of Theorem 1. It is invariant on the choice of an admissible metric on $X \times Y$ (see Remark 4).

Theorem 2 (i) ${ }^{s} r_{q}[F](\bar{x}, \bar{y}) \leq \overline{|\nabla F|_{q}^{\diamond}}(\bar{x}, \bar{y})$;

(ii) if $X$ and $Y$ are complete and gph $F$ is locally closed near $(\bar{x}, \bar{y})$, then ${ }^{s} r_{q}[F](\bar{x}, \bar{y})=$

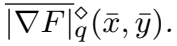

The estimate in the next proposition can be useful when formulating necessary conditions of $q$-subregularity.

Proposition 9 Suppose $X$ and $Y$ are normed spaces and $F$ is convex near $(\bar{x}, \bar{y})$. Then, $q \cdot{ }^{s} r_{q}[F](\bar{x}, \bar{y}) \leq \overline{|\partial F|}_{q}(\bar{x}, \bar{y})$.

Proof If ${ }^{s} r_{q}[F](\bar{x}, \bar{y})=0$, the conclusion is trivial. Suppose $0<\tau<{ }^{s} r_{q}[F](\bar{x}, \bar{y})$ and $0<\gamma<q$. Then, there exists a $\rho \in(0, q-\gamma)$ such that

$$
\tau d\left(x, F^{-1}(\bar{y})\right)<\|y-\bar{y}\|^{q}, \quad \forall x \in B_{\rho}(\bar{x}) \backslash F^{-1}(\bar{y}), y \in F(x) .
$$

Choose an arbitrary $(x, y) \in \operatorname{gph} F$ with $\|x-\bar{x}\|<\rho,\|y-\bar{y}\|<\rho, x \notin F^{-1}(\bar{y})$; $v^{*} \in J(y-\bar{y})$; and $x^{*} \in D^{*} F(x, y)\left(v^{*}+\xi(y) \rho \mathbb{B}^{*}\right)$. By (37), one can find a point $u \in F^{-1}(\bar{y})$ such that

$$
\tau\|x-u\|<\|y-\bar{y}\|^{q} .
$$

By the convexity of $F$, the Fréchet normal cone to its graph coincides with the normal cone in the sense of convex analysis and consequently, it holds

$$
\left\langle x^{*}, u-x\right\rangle \leq\left\langle v^{*}, \bar{y}-y\right\rangle+\xi(y) \rho\|y-\bar{y}\|=-(1-\xi(y) \rho)\|y-\bar{y}\| .
$$

Combining this with (38), we have

$$
\begin{aligned}
q\|y-\bar{y}\|^{q-1}\left\|x^{*}\right\|\|u-x\| & \geq-q\|y-\bar{y}\|^{q-1}\left\langle x^{*}, u-x\right\rangle \\
& \geq\left(q\|y-\bar{y}\|^{q-1}-\rho\right)\|y-\bar{y}\| \\
& >q\|y-\bar{y}\|^{q}-(q-\gamma)\|y-\bar{y}\| \\
& \geq q\|y-\bar{y}\|^{q}-(q-\gamma)\|y-\bar{y}\|^{q} \\
& =\gamma\|y-\bar{y}\|^{q}>\gamma \tau\|u-x\| .
\end{aligned}
$$

Hence,

$$
q\|y-\bar{y}\|^{q-1}\left\|x^{*}\right\|>\gamma \tau,
$$

and it follows from definitions (30) and (28) that $\overline{|\partial F|}_{q}(\bar{x}, \bar{y}) \geq \gamma \tau$. Passing to the limit in the last inequality as $\gamma \rightarrow q$ and $\tau \rightarrow{ }^{s} r_{q}[F](\bar{x}, \bar{y})$, we arrive at the claimed inequality. 
The next two corollaries summarize quantitative and qualitative criteria of Hölder metric subregularity of order $q$.

Corollary 3 (Quantitative criteria) Let $\gamma>0$. Consider the following conditions:

(a) $F$ is Hölder metrically subregular of order $q$ at $(\bar{x}, \bar{y})$ with some $\tau>0$;

(b) $\overline{|\nabla F|_{q}^{\diamond}}(\bar{x}, \bar{y})>\gamma$,

i.e., for some $\rho>0$ and any $(x, y) \in \operatorname{gph} F$ with $x \notin F^{-1}(\bar{y}), d(x, \bar{x})<\rho$, and $d(y, \bar{y})<\rho$, it holds $|\nabla F|_{q, \rho}^{\diamond}(x, y)>\gamma$, and consequently there is a $(u, v) \in \operatorname{gph} F$ such that

$$
(d(y, \bar{y}))^{q}-(d(v, \bar{y}))^{q}>\gamma d_{\rho}((u, v),(x, y)) ;
$$

(c) $\liminf _{\substack{x \rightarrow \bar{x} \\ x \notin F^{-1}(\bar{y}), y \in F(x)}} \frac{(d(y, \bar{y}))^{q}}{d(x, \bar{x})}>\gamma$;

(d) $\overline{|\nabla F|}_{q}(\bar{x}, \bar{y})>\gamma$,

i.e., for some $\rho>0$ and any $(x, y) \in \operatorname{gph} F$ with $x \notin F^{-1}(\bar{y}), d(x, \bar{x})<\rho$, and $d(y, \bar{y})<\rho$, it holds $q(d(y, \bar{y}))^{q-1}|\nabla F|_{\rho}(x, y)>\gamma$, and consequently, for any $\varepsilon>0$, there is $a(u, v) \in \operatorname{gph} F \cap B_{\varepsilon}(x, y)$ such that

$$
q(d(y, \bar{y}))^{q-1}(d(y, \bar{y})-d(v, \bar{y}))>\gamma d_{\rho}((u, v),(x, y)) ;
$$

(e) $\overline{\mid \nabla F}_{q}^{+}(\bar{x}, \bar{y})>\gamma$

i.e., for some $\rho>0$ and any $(x, y) \in X \times Y$ with $x \notin F^{-1}(\bar{y}), d(x, \bar{x})<\rho$, $d(y, \bar{y})<\rho$, and $(d(y, \bar{y}))^{q} / d(x, \bar{x}) \leq \gamma$, it holds $q(d(y, \bar{y}))^{q-1}|\nabla F|_{\rho}(x, y)>\gamma$ and consequently, for any $\varepsilon>0$, there is $a(u, v) \in \operatorname{gph} F \cap B_{\varepsilon}(x, y)$ such that (39) holds true;

(f) $X$ and $Y$ are normed spaces and $\overline{\mid \partial F}_{q}^{a}(\bar{x}, \bar{y})>\gamma$,

i.e., for some $\rho>0$ and any $(x, y) \in \operatorname{gph} F$ with $x \notin F^{-1}(\bar{y}),\|x-\bar{x}\|<\rho$, and $\|y-\bar{y}\|<\rho$, it holds

$$
q\|y-\bar{y}\|^{q-1}|\partial F|_{\xi(y) \rho}^{a}(x, y)>\gamma
$$

and consequently, there exists an $\varepsilon>0$ such that

$$
\begin{aligned}
& q\|y-\bar{y}\|^{q-1}\left\|x^{*}\right\|>\gamma \text { for all } \\
& \qquad x^{*} \in D^{*} F(x, y)\left(J\left(B_{\varepsilon}(y-\bar{y})\right)+\xi(y) \rho \mathbb{B}^{*}\right) ;
\end{aligned}
$$

(g) $X$ and $Y$ are normed spaces and $\overline{|\partial F|}_{q}^{a+}(\bar{x}, \bar{y})>\gamma$,

i.e., for some $\rho>0$ and any $(x, y) \in X \times Y$ with $x \notin F^{-1}(\bar{y}),\|x-\bar{x}\|<\rho$, $\|y-\bar{y}\|<\rho$, and $\|y-\bar{y}\|^{q} /\|x-\bar{x}\| \leq \gamma$, condition (40) holds and consequently, there exists an $\varepsilon>0$ such that (41) holds true;

(h) $X$ and $Y$ are normed spaces and $\overline{|\partial F|}_{q}(\bar{x}, \bar{y})>\gamma$,

i.e., for some $\rho>0$ and any $(x, y) \in \operatorname{gph} F$ with $x \notin F^{-1}(\bar{y}),\|x-\bar{x}\|<\rho$, and $\|y-\bar{y}\|<\rho$, it holds

$$
q\|y-\bar{y}\|^{q-1}|\partial F|_{\xi(y) \rho}(x, y)>\gamma
$$

and consequently,

$$
\begin{aligned}
q\|y-\bar{y}\|^{q-1}\left\|x^{*}\right\|>\gamma \text { for all } \\
\qquad x^{*} \in D^{*} F(x, y)\left(J(y-\bar{y})+\xi(y) \rho \mathbb{B}^{*}\right)
\end{aligned}
$$


(i) $X$ and $Y$ are normed spaces and $\overline{\mid \partial F}_{q}^{+}(\bar{x}, \bar{y})>\gamma$, i.e., for some $\rho>0$ and any $(x, y) \in X \times Y$ with $x \notin F^{-1}(\bar{y}),\|x-\bar{x}\|<\rho$, $\|y-\bar{y}\|<\rho$, and $\|y-\bar{y}\|^{q} /\|x-\bar{x}\| \leq \gamma$, condition (42) holds, and consequently (43) holds true;

(j) $X$ and $Y$ are finite dimensional normed spaces and

$$
\left\|x^{*}\right\|>\gamma \quad \text { for all } x^{*} \in \bar{D}_{q}^{*>} F(\bar{x}, \bar{y})\left(\mathbb{S}_{Y^{*}}^{*}\right) .
$$

The following implications hold true:

(i) (c) $\Rightarrow$ (e), (d) $\Rightarrow$ (e), (e) $\Rightarrow$ (b), (f) $\Rightarrow$ (g) $\Rightarrow$ (i), (f) $\Rightarrow$ (h) $\Rightarrow$ (i);

(ii) if $\gamma<\tau$, then (a) $\Rightarrow$ (b);

(iii) if $\tau \leq \gamma, X$ and $Y$ are complete, and gph $F$ is locally closed near $(\bar{x}, \bar{y})$, then (b) $\Rightarrow(\mathrm{a})$.

Suppose $X$ and $Y$ are normed spaces.

(iv) If $F$ is convex, and $\gamma<q \tau$, then (a) $\Rightarrow(\mathrm{h}$ );

(v) (f) $\Rightarrow$ (d) and (g) $\Rightarrow$ (e)

provided that $X$ and $Y$ are Asplund and gph $F$ is locally closed near $(\bar{x}, \bar{y})$;

(vi) $(\mathrm{h}) \Leftrightarrow(\mathrm{d})$ and $(\mathrm{i}) \Leftrightarrow(\mathrm{e})$ provided that $Y$ is Fréchet smooth and one of the following conditions is satisfied:

(a) $X$ is Asplund and gph $F$ is locally closed near $(\bar{x}, \bar{y})$;

(b) $F$ is convex near $(\bar{x}, \bar{y})$;

(vii) (b) $\Leftrightarrow$ (d) $\Leftrightarrow$ (e) $\Leftrightarrow$ (h) $\Leftrightarrow$ (i) provided that $F$ is convex near $(\bar{x}, \bar{y})$ and $q=1$;

(viii) if $X$ and $Y$ are finite dimensional normed spaces, then $(\mathrm{f}) \Leftrightarrow(\mathrm{h}) \Leftrightarrow(\mathrm{j})$.

The conclusions of Corollary 3 are illustrated in Fig. 3.

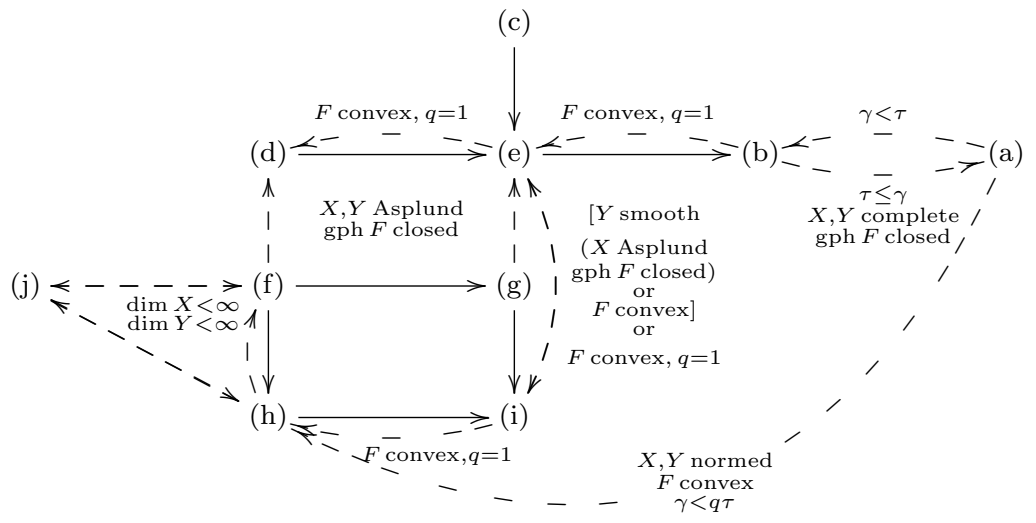

Fig. 3 Corollary 3

Corollary 4 (Qualitative criteria) Suppose $X$ and $Y$ are complete metric spaces and gph $F$ is locally closed near $(\bar{x}, \bar{y})$. Then, $F$ is Hölder metrically subregular of order $q$ at $(\bar{x}, \bar{y})$ provided that one of the following conditions holds true: 
(a) $\overline{|\nabla F|}_{q}^{\diamond}(\bar{x}, \bar{y})>0$

(b) $\liminf _{\substack{x \rightarrow \bar{x} \\ x \notin F^{-1}(\bar{y}), y \in F(x)}} \frac{(d(y, \bar{y}))^{q}}{d(x, \bar{x})}>0$;

(c) $\overline{|\nabla F|}_{q}(\bar{x}, \bar{y})>0$;

(d) $\left.\overline{|\nabla F|}\right|_{q} ^{+}(\bar{x}, \bar{y})>0$, or equivalently,

$$
\lim _{\rho \downarrow 0} \inf _{d(x, \bar{x})<\rho, d(y, \bar{y})<\rho, \frac{(d(y, \bar{y}))^{q}}{d(x, \bar{x})}<\rho}(d(y, \bar{y}))^{q-1}|\nabla F|_{\rho}(x, y)>0 .
$$

If $X$ and $Y$ are Asplund spaces, then the following conditions are also sufficient:

(e) $\overline{|\partial F|}_{q}^{a}(\bar{x}, \bar{y})>0$;

(f) $\overline{|\partial F|}_{q}^{a+}(\bar{x}, \bar{y})>0$, or equivalently,

$$
\lim _{\rho \downarrow 0} \inf _{\substack{\|x-\bar{x}\|<\rho,\|y-\bar{y}\|<\rho, \frac{\|y-\bar{y}\|^{q}}{\|x-\bar{x}\|}<\rho \\(x, y) \in \operatorname{gph} F, x \notin F^{-1}(\bar{y})}}\|y-\bar{y}\|^{q-1}|\partial F|_{\xi(y) \rho}^{a}(x, y)>0 .
$$

The next two conditions:

(g) $\overline{|\partial F|}_{q}(\bar{x}, \bar{y})>0$;

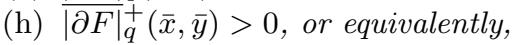

$$
\lim _{\rho \downarrow 0} \inf _{\substack{\|x-\bar{x}\|<\rho,\|y-\bar{y}\|<\rho, \frac{\|y-\bar{y}\|^{q}}{\|x-\bar{x}\|}<\rho \\(x, y) \in \operatorname{gph} F, x \notin F^{-1}(\bar{y})}}\|y-\bar{y}\|^{q-1}|\partial F|_{\xi(y) \rho}(x, y)>0,
$$

are sufficient provided that $X$ and $Y$ are Banach spaces and one of the following conditions is satisfied:

- $X$ is Asplund and $Y$ is Fréchet smooth,

- $F$ is convex near $(\bar{x}, \bar{y})$ and either $Y$ is Fréchet smooth or $q=1$.

If $X$ and $Y$ are finite dimensional normed spaces, then the following condition is also sufficient:

(i) $0 \notin \bar{D}_{q}^{*>} F(\bar{x}, \bar{y})\left(\mathbb{S}_{Y^{*}}^{*}\right)$.

\section{Moreover,}

(i) condition (a) is also necessary for the metric q-subregularity of $F$ at $(\bar{x}, \bar{y})$;

(ii) (b) $\Rightarrow$ (d), (c) $\Rightarrow$ (d), (d) $\Rightarrow$ (a), (e) $\Rightarrow$ (f), (g) $\Rightarrow$ (h).

Suppose $X$ and $Y$ are Banach spaces.

(iii) If $X$ and $Y$ are Asplund, then (e) $\Rightarrow$ (c) and (f) $\Rightarrow$ (d);

(iv) if $Y$ is Fréchet smooth and either $X$ is Asplund or $F$ is convex near $(\bar{x}, \bar{y})$, then (e) $\Leftrightarrow$ (c) and (f) $\Leftrightarrow(\mathrm{d})$;

(v) if $F$ is convex near $(\bar{x}, \bar{y})$, then condition (g) is also necessary for the metric q-subregularity of $F$ at $(\bar{x}, \bar{y})$;

(vi) if $F$ is convex near $(\bar{x}, \bar{y})$ and $q=1$, then $(\mathrm{a}) \Leftrightarrow(\mathrm{c}) \Leftrightarrow(\mathrm{d}) \Leftrightarrow(\mathrm{g}) \Leftrightarrow(\mathrm{h})$;

(vii) if $X$ and $Y$ are finite dimensional normed spaces, then (e) $\Leftrightarrow(\mathrm{g}) \Leftrightarrow$ (i). 


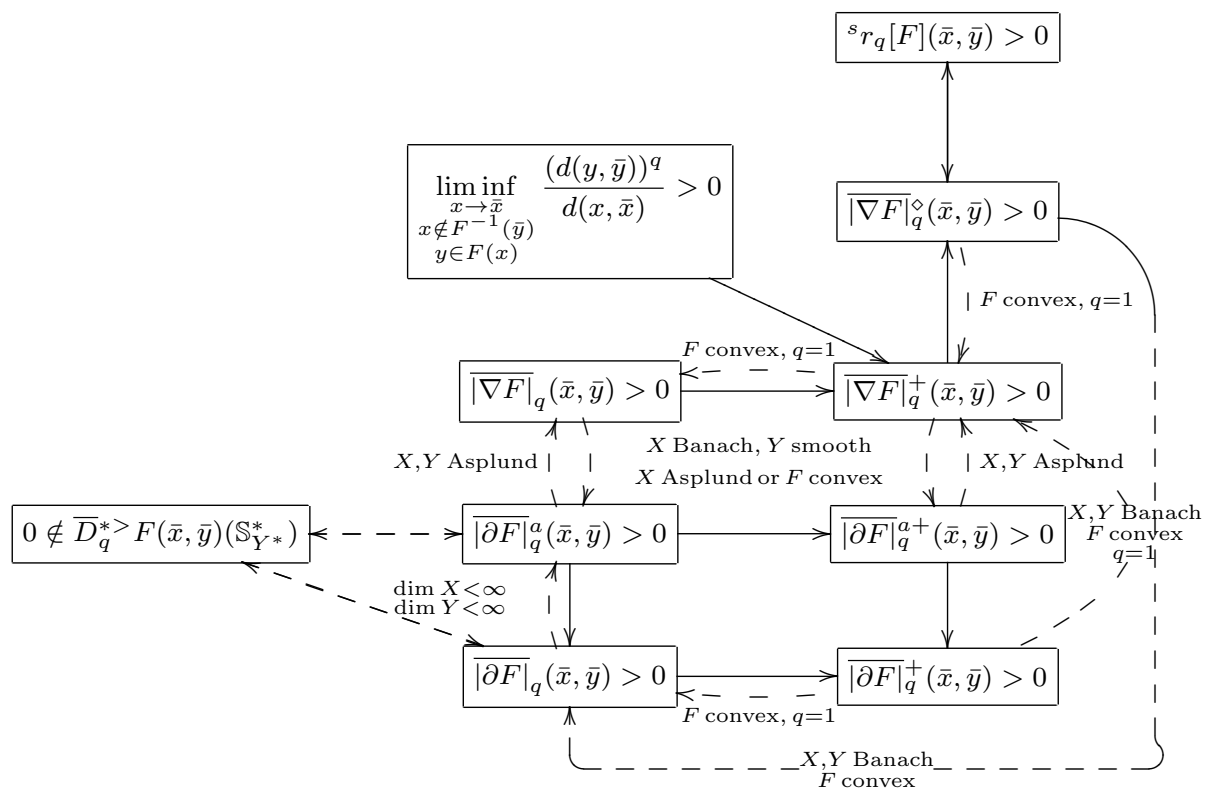

Fig. 4 Corollary 4

The conclusions of Corollary 4 are illustrated in Fig. 4.

Another three subdifferential criteria of Hölder metric subregularity of order $q$ have been established recently by $\mathrm{Li}$ and Mordukhovich [34] (cf. [40]). In the case when $Y$ is a Banach space, they introduced two modifications of the duality mapping $J$ defined by (5), namely, the $q$-duality mapping $J^{q}$ and, given an $\varepsilon \geq 0$, its normalized $\varepsilon$-enlargement $J_{\varepsilon}^{q}$ (both acting $Y \backslash\{0\} \rightrightarrows Y^{*}$ ):

$$
\begin{gathered}
J^{q}(y):=q\|y\|^{q-1} J(y), \\
J_{\varepsilon}^{q}(y):=\left\{\frac{y^{*}+\varepsilon v^{*}}{\left\|y^{*}+\varepsilon v^{*}\right\|} \mid y^{*} \in J^{q}(y),\left\|v^{*}\right\| \leq 1, y^{*}+\varepsilon v^{*} \neq 0\right\} .
\end{gathered}
$$

Using (44), the authors defined for the mapping $F$ two nonnegative constants:

$$
\begin{gathered}
\alpha:=\sup _{\varepsilon>0} \inf \left\{q\left\|x^{*}\right\| \cdot\left\|y^{\prime}-\bar{y}\right\|^{q-1} \mid(x, y) \in \operatorname{gph} F, x \notin F^{-1}(\bar{y}),\right. \\
\|x-\bar{x}\|<\varepsilon,\|y-\bar{y}\|<\min \left\{\varepsilon,\|x-\bar{x}\|^{\frac{1}{2}}\right\}, \\
\left.\left\|y^{\prime}-y\right\|<\|x-\bar{x}\|^{\frac{1}{q}}, y^{\prime} \neq \bar{y}, x^{*} \in D^{*} F(x, y)\left(J_{\varepsilon}^{q}\left(y^{\prime}-\bar{y}\right)\right)\right\}, \\
\beta:=\sup _{\varepsilon>0} \inf \left\{q\left\|x^{*}\right\| \cdot\|y-\bar{y}\|^{q-1} \mid(x, y) \in \operatorname{gph} F, x \notin F^{-1}(\bar{y}),\right. \\
\|x-\bar{x}\|<\varepsilon,\|y-\bar{y}\|<\min \left\{\varepsilon,\|x-\bar{x}\|^{\frac{1}{2}}\right\}, \\
\left.x^{*} \in D^{*} F(x, y)\left(J_{\varepsilon}^{q}(y-\bar{y})\right)\right\},
\end{gathered}
$$

which played a crucial role when determining the Hölder metric subregularity of $F$. 
Using the notation adopted in the current article and with $\alpha$ and $\beta$ defined by (45) and (46), respectively, [34, Theorems 3.3, 5.1, and 5.3] can be formulated in the following way.

Theorem 3 (i) Suppose $X$ and $Y$ are Asplund and gph $F$ is locally closed near $(\bar{x}, \bar{y})$. Then, 1) $\alpha \leq{ }^{s} r_{q}[F](\bar{x}, \bar{y})$ and 2) condition $\alpha>0$ is sufficient for Hölder metric subregularity of order $q$ of $F$ at $(\bar{x}, \bar{y})$.

(ii) Suppose $X$ is Asplund, $Y$ is Fréchet smooth, and gph $F$ is locally closed near $(\bar{x}, \bar{y})$. Then, 1) $\beta \leq{ }^{s} r_{q}[F](\bar{x}, \bar{y})$ and 2) condition $\beta>0$ is sufficient for Hölder metric subregularity of order $q$ of $F$ at $(\bar{x}, \bar{y})$.

(iii) Suppose $X$ is Banach, $Y$ is Fréchet smooth, and gph $F$ is closed and convex. Then, condition $\beta>0$ is necessary and sufficient for Hölder metric subregularity of order $q$ of $F$ at $(\bar{x}, \bar{y})$.

The assertions of the above theorem are obvious consequences of Proposition 7 and Corollary 3 thanks to the next proposition.

Proposition 10 Suppose $X$ and $Y$ are normed spaces. Then,

(i) $\alpha \leq \overline{\mid \partial F}_{q}^{+a}(\bar{x}, \bar{y})$,

(ii) $\overline{|\partial F|}_{q}(\bar{x}, \bar{y}) \leq \beta \leq \overline{\mid \partial F}_{q}^{+}(\bar{x}, \bar{y})$.

Proof (i) If $\overline{|\partial F|}_{q}^{+a}(\bar{x}, \bar{y})=\infty$, the inequality is satisfied trivially. Suppose $\overline{|\partial F|}_{q}^{+a}(\bar{x}, \bar{y})<$ $\gamma<\infty$. Then, by definition (33),

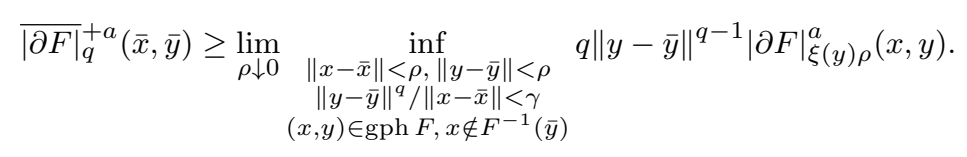

If $0<\rho<\gamma^{-\frac{1}{1-q / 2}}, 0<\|x-\bar{x}\|<\rho$, and $\|y-\bar{y}\|^{q} /\|x-\bar{x}\|<\gamma$, then,

$$
\|y-\bar{y}\|<(\gamma\|x-\bar{x}\|)^{\frac{1}{q}}=\|x-\bar{x}\|^{\frac{1}{2}}\left(\gamma\|x-\bar{x}\|^{1-\frac{q}{2}}\right)^{\frac{1}{q}}<\|x-\bar{x}\|^{\frac{1}{2}}
$$

Hence,

$$
\overline{\mid \partial F}_{q}^{+a}(\bar{x}, \bar{y}) \geq \lim _{\rho \downarrow 0} \inf _{\substack{\|x-\bar{x}\|<\rho,\|y-\bar{y}\|<\rho \\\|y-\bar{y}\|<\|x-\bar{x}\|^{\frac{1}{2}} \\(x, y) \in \operatorname{gph} F, x \notin F^{-1}(\bar{y})}} q\|y-\bar{y}\|^{q-1}|\partial F|_{\xi(y) \rho}^{a}(x, y) .
$$

If $(x, y) \in \operatorname{gph} F, y \neq \bar{y}$, and $\varepsilon \in(0,\|y-\bar{y}\|)$, then, by definition (29), it holds

$$
\begin{aligned}
& |\partial F|_{\xi(y) \rho}^{a}(x, y) \geq \inf _{x^{*} \in D^{*} F(x, y)\left(J(v)+\xi(y) \rho \mathbb{B}^{*}\right)}\left\|x^{*}\right\| \\
& =\inf _{\substack{\left\|y^{\prime}-y\right\|<\varepsilon \\
x^{*} \in D^{*} F(x, y)\left(J\left(y^{\prime}-\bar{y}\right)+\xi(y) \rho \mathbb{B}^{*}\right)}}\left\|x^{*}\right\| \\
& =\inf _{\substack{\left\|y^{\prime}-y\right\|<\varepsilon \\
x^{*} \in D^{*} F(x, y)\left(y^{*}+\xi(y) \rho v^{*}\right)}}\left\|x^{*}\right\| . \\
& y^{*} \in J\left(y^{\prime}-\bar{y}\right),\left\|v^{*}\right\| \leq 1
\end{aligned}
$$


Let $\rho \in(0,1),(x, y) \in \operatorname{gph} F, x \neq \bar{x}$, and $y \neq \bar{y}$ be such that $\|y-\bar{y}\|^{1-q} \leq q$. Choose an $\varepsilon \in(0,\|y-\bar{y}\|)$ such that inequality $\left\|y^{\prime}-y\right\|<\varepsilon$ implies the following estimates:

$$
\left\|y^{\prime}-y\right\|<\|x-\bar{x}\|^{\frac{1}{q}}, \quad 1-\rho<\left(\left\|y^{\prime}-\bar{y}\right\| /\|y-\bar{y}\|\right)^{q-1}<2 .
$$

Then, given any $y^{\prime} \in Y$ with $\left\|y^{\prime}-y\right\|<\varepsilon, y^{*} \in J\left(y^{\prime}-\bar{y}\right)$, and $\left\|v^{*}\right\| \leq 1$, we have

$$
\begin{gathered}
\left\|y^{*}+\xi(y) \rho v^{*}\right\|>1-\rho>0, \\
\rho^{\prime}:=\rho\left(\left\|y^{\prime}-\bar{y}\right\| /\|y-\bar{y}\|\right)^{q-1}<2 \rho, \\
y^{*}+\xi(y) \rho v^{*}=y^{*}+\frac{\|y-\bar{y}\|^{1-q}}{q} \rho v^{*}=\frac{\left\|y^{\prime}-\bar{y}\right\|^{1-q}}{q}\left(q\left\|y^{\prime}-\bar{y}\right\|^{q-1} y^{*}+\rho^{\prime} v^{*}\right), \\
\frac{y^{*}+\xi(y) \rho v^{*}}{\left\|y^{*}+\xi(y) \rho v^{*}\right\|}=\frac{q\left\|y^{\prime}-\bar{y}\right\|^{q-1} y^{*}+\rho^{\prime} v^{*}}{\|q\| y^{\prime}-\bar{y}\left\|^{q-1} y^{*}+\rho^{\prime} v^{*}\right\|} \in J_{\rho^{\prime}}^{q}\left(y^{\prime}-\bar{y}\right) \subset J_{2 \rho}^{q}\left(y^{\prime}-\bar{y}\right) .
\end{gathered}
$$

Hence,

$$
\begin{aligned}
D^{*} F(x, y)\left(y^{*}+\xi(y) \rho v^{*}\right) & =\left\|y^{*}+\xi(y) \rho v^{*}\right\| D^{*} F(x, y)\left(\frac{y^{*}+\xi(y) \rho v^{*}}{\left\|y^{*}+\xi(y) \rho v^{*}\right\|}\right) \\
& \subset\left\|y^{*}+\xi(y) \rho v^{*}\right\| D^{*} F(x, y)\left(J_{2 \rho}^{q}\left(y^{\prime}-\bar{y}\right)\right),
\end{aligned}
$$

and consequently,

$$
|\partial F|_{\xi(y) \rho}^{a}(x, y) \geq(1-\rho) \underset{\substack{\left\|y^{\prime}-y\right\|<\|x-\bar{x}\|^{\frac{1}{q}}, y^{\prime} \neq \bar{y} \\ x^{*} \in D^{*} F(x, y)\left(J_{2 \rho}^{q}\left(y^{\prime}-\bar{y}\right)\right)}}{\inf ^{*}\left\|x^{*}\right\| .}
$$

Combining this estimate with (47) and making use of (48), we obtain

$$
\begin{aligned}
& \overline{\mid \partial F}_{q}^{+a}(\bar{x}, \bar{y}) \geq \lim _{\rho \downarrow 0}(1-\rho)^{2} \underset{\substack{\|x-\bar{x}\|<\rho,\|y-\bar{y}\|<\rho \\
\|y-\bar{y}\|<\|x-\bar{x}\|^{\frac{1}{2}}}}{\inf _{(x, y)}} q\left\|y^{\prime}-\bar{y}\right\|^{q-1} \underset{\substack{\left\|y^{\prime}-y\right\|<\|x-\bar{x}\|^{\frac{1}{q}}, y^{\prime} \neq \bar{y} \\
x^{*} \in D^{*} F(x, y)\left(J_{2 \rho}^{q}\left(y^{\prime}-\bar{y}\right)\right)}}{\left\|x^{*}\right\|}
\end{aligned}
$$

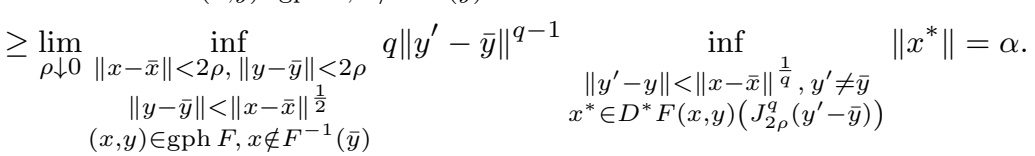

(ii) As in the proof of (i), we have from definition (32)

$$
{\overline{|\partial F|_{q}^{+}}}^{+}(\bar{x}, \bar{y}) \geq \lim _{\rho \downarrow 0} \inf _{\substack{\|x-\bar{x}\|<\rho,\|y-\bar{y}\|<\rho \\\|y-\bar{y}\|<\|x-\bar{x}\|^{\frac{1}{2}} \\(x, y) \in \operatorname{gph} F, x \notin F^{-1}(\bar{y})}} q\|y-\bar{y}\|^{q-1}|\partial F|_{\xi(y) \rho}(x, y),
$$

where, by definition (28) and using again (simplified versions of) the same arguments as in the proof of (i),

$$
|\partial F|_{\xi(y) \rho}(x, y)=\inf _{x^{*} \in D^{*} F(x, y)\left(y^{*}+\xi(y) \rho v^{*}\right)}\left\|x^{*}\right\| \geq(1-\rho) \inf _{y^{*} \in J(y-\bar{y}),\left\|v^{*}\right\| \leq 1} \inf _{x^{*} F(x, y)\left(J_{\rho}^{q}(y-\bar{y})\right)}\left\|x^{*}\right\| .
$$

The second inequality in (ii) follows from the last two estimates and definition (46). 
Let $0<\varepsilon<q^{\frac{1}{2-q}},(x, y) \in \operatorname{gph} F, 0<\|y-\bar{y}\|<\varepsilon$, and $w^{*} \in J_{\varepsilon}^{q}(y-\bar{y})$, that is,

$$
w^{*}=\frac{q\|y-\bar{y}\|^{q-1} y^{*}+\varepsilon v^{*}}{\|q\| y-\bar{y}\left\|^{q-1} y^{*}+\varepsilon v^{*}\right\|}
$$

for some $y^{*} \in J(y-\bar{y})$ and $v^{*} \in \mathbb{B}^{*}$. Observe that $\xi(y)=\|y-\bar{y}\|^{1-q} / q<\varepsilon^{1-q} / q$,

$$
\begin{gathered}
\|q\| y-\bar{y}\left\|^{q-1} y^{*}+\varepsilon v^{*}\right\| \geq q \varepsilon^{q-1}-\varepsilon=\varepsilon^{q-1}\left(q-\varepsilon^{2-q}\right)>0, \\
q\|y-\bar{y}\|^{q-1} y^{*}+\varepsilon v^{*}=q\|y-\bar{y}\|^{q-1}\left(y^{*}+\varepsilon \xi(y) v^{*}\right) .
\end{gathered}
$$

Hence,

$$
\begin{aligned}
\left\|w^{*}-y^{*}\right\| & =\left\|\frac{y^{*}+\varepsilon \xi(y) v^{*}}{\left\|y^{*}+\varepsilon \xi(y) v^{*}\right\|}-y^{*}\right\|=\frac{\left\|y^{*}\left(1-\left\|y^{*}+\varepsilon \xi(y) v^{*}\right\|\right)+\varepsilon \xi(y) v^{*}\right\|}{\left\|y^{*}+\varepsilon \xi(y) v^{*}\right\|} \\
& \leq \frac{\left|1-\left\|y^{*}+\varepsilon \xi(y) v^{*}\right\|\right|+\varepsilon \xi(y)}{1-\varepsilon \xi(y)} \leq \frac{2 \varepsilon \xi(y)}{1-\varepsilon \xi(y)} \leq \frac{2 \varepsilon}{1-\varepsilon^{2-q} / q} \xi(y) .
\end{aligned}
$$

Given a $\rho>0$, one can always choose an $\varepsilon>0$ such that $2 \varepsilon /\left(1-\varepsilon^{2-q} / q\right)<\rho$, and consequently $D^{*} F(x, y)\left(w^{*}\right) \subset D^{*} F(x, y)\left(y^{*}+\xi(y) \rho \mathbb{B}^{*}\right)$. The first inequality follows from definitions (30), (28), and (46).

Proof of Theorem 3 The conclusions follow from Theorem 2(ii) thanks to Propositions 7 and 10.

(i) requires parts (ii) and (iv) of Proposition 7 and part (i) of Proposition 10.

(ii) requires parts (ii) and (v)(a) of Proposition 7 and part (ii) of Proposition 10.

(iii) requires parts (ii) and (v)(b) of Proposition 7, part (ii) of Proposition 10, and, additionally, Proposition 9.

Remark 8 Assertions (i) and (ii) of Theorem 3 are in general weaker than the corresponding ones in Corollary 3. They can be strengthened if inequality $\|y-\bar{y}\|<$ $\min \left\{\varepsilon,\|x-\bar{x}\|^{\frac{1}{2}}\right\}$ in definitions (45) and (46) is replaced by a more restrictive (when $\|x-\bar{x}\|<1)$ one: $\|y-\bar{y}\|<\min \left\{\varepsilon,\|x-\bar{x}\|^{\frac{1}{p}}\right\}$ where $p$ can be any number in the interval $(q, 2)$. Proposition 10 remains true in this situation. The only change required in its proof is replacing inequality $\rho<\gamma^{-\frac{1}{1-q / 2}}$ by the following one: $\rho<\gamma^{-\frac{1}{1-q / p}}$.

The next example illustrates the computation of the constants involved in the definition and characterizations of Hölder metric subregularity.

Example 1 Consider a mapping $F: \mathbb{R} \rightarrow \mathbb{R}$ given by

$$
F(x):=\left(x_{+}\right)^{2}= \begin{cases}x^{2} & \text { if } x \geq 0 \\ 0 & \text { otherwise }\end{cases}
$$

cf. [34, Example 3.8]. It is obviously Hölder metrically subregular of order $q=1 / 2$ at $(0,0)$. Note that $F^{-1}(0)=(-\infty, 0]$ and, if $x>0$, then $d\left(x, F^{-1}(0)\right)=x$ and $d(0, F(x))=x^{2}$. This allows us to compute the modulus of Hölder metric subregularity (21):

$$
{ }^{s} r_{q}[F](0,0)=\liminf _{x \downarrow 0} \frac{\left(x^{2}\right)^{\frac{1}{2}}}{x}=1 .
$$


This result can also be deduced from Theorem 2(ii). For that, one needs to compute the uniform strict $q$-slope (24). Let $x>0, y=x^{2}$, and $\rho>0$. Then the nonlocal $(q, \rho)$ slope $(23)$ of $F$ at $(x, y)$ takes the following form:

$$
|\nabla F|_{q, \rho}^{\diamond}(x, y)=\sup _{u \neq x} \frac{\left[x-u_{+}\right]_{+}}{\max \left\{|u-x|, \rho\left|u_{+}^{2}-x^{2}\right|\right\}} .
$$

If $u>x$, then $x-u_{+}=x-u<0$ and the expression under sup in the right-hand side of (49) equals 0 . If $u \leq 0$, it equals $x / \max \left\{|u-x|, \rho x^{2}\right\}$ and is continuous and increasing on $(-\infty, 0]$ as a function of $u$, attaining its maximum at 0 . Hence,

$$
|\nabla F|_{q, \rho}^{\diamond}(x, y)=\sup _{0 \leq u<x} \frac{x-u}{\max \left\{x-u, \rho\left(x^{2}-u^{2}\right)\right\}}=\frac{1}{\max \{1, \rho x\}},
$$

and consequently $|\nabla F|_{q, \rho}^{\diamond}(x, y)=1$ if $\rho x \leq 1$. It follows immediately from definition (24) that $\left.\overline{|\nabla F|}\right|_{q}(0,0)=1$.

Hölder metric subregularity of $F$ can also be established from the estimates in Proposition 7 after computing any of the local strict $q$-slopes (26), (27), (30)-(33) which, in turn, depend on local $\rho$-slopes (25), (28), and (29). Observe that the last three constants do not depend on $q$.

Similarly to the above, for $x>0, y=x^{2}$, and $\rho>0$, one has

$$
\begin{aligned}
|\nabla F|_{\rho}(x, y) & =\limsup _{u \rightarrow x, u \neq x} \frac{\left[x^{2}-u^{2}\right]_{+}}{\max \left\{|u-x|, \rho\left|u^{2}-x^{2}\right|\right\}} \\
& =\lim _{u \uparrow x} \frac{x+u}{\max \{1, \rho(x+u)\}}=\frac{2 x}{\max \{1,2 \rho x\}}
\end{aligned}
$$

and consequently, $|\nabla F|_{\rho}(x, y)=2 x$ if $\rho x \leq 1 / 2$. Mapping $F$ is differentiable and $D^{*} F(x, y)\left(y^{*}\right)=\left\{2 x y^{*}\right\}$ for any $x \geq 0, y=x^{2}$, and $y^{*} \in \mathbb{R}$. Duality mapping (5) in $\mathbb{R}$ has a simple representation: $J(v)=1$ if $v>0$ and $J(v)=-1$ if $v<0$. Thus,

$$
|\partial F|_{\rho}(x, y)=|\partial F|_{\rho}^{a}(x, y)=\inf _{x^{*} \in D^{*} F(x, y)\left(1+\rho \mathbb{B}^{*}\right)}\left|x^{*}\right|=2 x(1-\rho) .
$$

Observing that $(d(y, 0))^{q-1}=1 / x$ and $\xi(y)=2 x$ in (30)-(33), we arrive at

$$
\begin{aligned}
\overline{|\nabla F|}_{q}(\bar{x}, \bar{y}) & =\overline{|\nabla F|}_{q}^{+}(\bar{x}, \bar{y}) \\
& =\overline{|\partial F|}_{q}(\bar{x}, \bar{y})=\overline{|\partial F|}_{q}^{+}(\bar{x}, \bar{y})=\overline{|\partial F|}_{q}^{a}(\bar{x}, \bar{y})=\overline{\mid \partial F}_{q}^{+a}(\bar{x}, \bar{y})=1 .
\end{aligned}
$$

By Proposition 7, this ensures that $F$ is Hölder metrically subregular of order $\frac{1}{2}$ at $(0,0)$ with modulus not less than 1 .

\section{References}

1. Apetrii, M., Durea, M., Strugariu, R.: On subregularity properties of set-valued mappings. Set-Valued Var. Anal. 21(1), 93-126 (2013)

2. Azé, D.: A survey on error bounds for lower semicontinuous functions. In: Proceedings of 2003 MODE-SMAI Conference, ESAIM Proc., vol. 13, pp. 1-17. EDP Sci., Les Ulis (2003)

3. Azé, D.: A unified theory for metric regularity of multifunctions. J. Convex Anal. 13(2), 225-252 (2006)

4. Azé, D., Corvellec, J.N.: Characterizations of error bounds for lower semicontinuous functions on metric spaces. ESAIM Control Optim. Calc. Var. 10(3), 409-425 (2004)

5. Borwein, J.M., Zhu, Q.J.: Techniques of Variational Analysis. Springer, New York (2005) 
6. Borwein, J.M., Zhuang, D.M.: Verifiable necessary and sufficient conditions for openness and regularity for set-valued and single-valued maps. J. Math. Anal. Appl. 134, 441-459 (1988)

7. Corvellec, J.N., Motreanu, V.V.: Nonlinear error bounds for lower semicontinuous functions on metric spaces. Math. Program., Ser. A 114(2), 291-319 (2008)

8. De Giorgi, E., Marino, A., Tosques, M.: Problems of evolution in metric spaces and maximal decreasing curve. Atti Accad. Naz. Lincei Rend. Cl. Sci. Fis. Mat. Natur. (8) 68(3), 180-187 (1980). In Italian

9. Dontchev, A.L., Rockafellar, R.T.: Regularity and conditioning of solution mappings in variational analysis. Set-Valued Anal. 12(1-2), 79-109 (2004)

10. Dontchev, A.L., Rockafellar, R.T.: Implicit Functions and Solution Mappings. A View from Variational Analysis. Springer Monographs in Mathematics. Springer, Dordrecht (2009)

11. Ekeland, I.: On the variational principle. J. Math. Anal. Appl. 47, 324-353 (1974)

12. Fabian, M.: Subdifferentiability and trustworthiness in the light of a new variational principle of Borwein and Preiss. Acta Univ. Carolinae 30, 51-56 (1989)

13. Fabian, M.J., Henrion, R., Kruger, A.Y., Outrata, J.V.: Error bounds: necessary and sufficient conditions. Set-Valued Var. Anal. 18(2), 121-149 (2010)

14. Fabian, M.J., Henrion, R., Kruger, A.Y., Outrata, J.V.: About error bounds in metric spaces. In: D. Klatte, H.J. Lüthi, K. Schmedders (eds.) Operations Research Proceedings 2011. Selected papers of the Int. Conf. Operations Research (OR 2011), August 30 September 2, 2011, Zurich, Switzerland, pp. 33-38. Springer-Verlag, Berlin (2012)

15. Frankowska, H.: An open mapping principle for set-valued maps. J. Math. Anal. Appl. 127(1), 172-180 (1987)

16. Frankowska, H.: High order inverse function theorems. Ann. Inst. H. Poincaré Anal. Non Linéaire 6(suppl.), 283-303 (1989)

17. Frankowska, H., Quincampoix, M.: Hölder metric regularity of set-valued maps. Math. Program. 132(1-2, Ser. A), 333-354 (2012)

18. Gaydu, M., Geoffroy, M.H., Jean-Alexis, C.: Metric subregularity of order $q$ and the solving of inclusions. Cent. Eur. J. Math. 9(1), 147-161 (2011)

19. Henrion, R., Jourani, A.: Subdifferential conditions for calmness of convex constraints. SIAM J. Optim. 13(2), 520-534 (2002)

20. Henrion, R., Jourani, A., Outrata, J.: On the calmness of a class of multifunctions. SIAM J. Optim. 13(2), 603-618 (2002)

21. Henrion, R., Outrata, J.V.: A subdifferential condition for calmness of multifunctions. J. Math. Anal. Appl. 258(1), 110-130 (2001)

22. Ioffe, A.D.: Regular points of Lipschitz functions. Trans. Amer. Math. Soc. 251, 61-69 $(1979)$

23. Ioffe, A.D.: On subdifferentiability spaces. Ann. New York Acad. Sci. 410, 107-119 (1983)

24. Ioffe, A.D.: Metric regularity and subdifferential calculus. Russian Math. Surveys 55, 501-558(2000)

25. Ioffe, A.D.: Nonlinear regularity models. Math. Program. 139(1-2), 223-242 (2013)

26. Ioffe, A.D., Outrata, J.V.: On metric and calmness qualification conditions in subdifferential calculus. Set-Valued Anal. 16(2-3), 199-227 (2008)

27. Ioffe, A.D., Tikhomirov, V.M.: Theory of Extremal Problems, Studies in Mathematics and its Applications, vol. 6. North-Holland Publishing Co., Amsterdam (1979)

28. Klatte, D.: On quantitative stability for non-isolated minima. Control Cybernet. 23(1-2), 183-200 (1994)

29. Klatte, D., Kruger, A.Y., Kummer, B.: From convergence principles to stability and optimality conditions. J. Convex Anal. 19(4), 1043-1072 (2012)

30. Kruger, A.Y.: On Fréchet subdifferentials. J. Math. Sci. 116(3), 3325-3358 (2003)

31. Kruger, A.Y.: Error bounds and metric subregularity. Optimization (2014). DOI 10.1080/02331934.2014.938074

32. Kummer, B.: Inclusions in general spaces: Hoelder stability, solution schemes and Ekeland's principle. J. Math. Anal. Appl. 358(2), 327-344 (2009)

33. Leventhal, D.: Metric subregularity and the proximal point method. J. Math. Anal. Appl. 360(2), 681-688 (2009)

34. Li, G., Mordukhovich, B.S.: Hölder metric subregularity with applications to proximal point method. SIAM J. Optim. 22(4), 1655-1684 (2012)

35. Lucchetti, R.: Convexity and Well-Posed Problems. CMS Books in Mathematics/Ouvrages de Mathématiques de la SMC, 22. Springer, New York (2006) 
36. Mordukhovich, B.S.: Variational Analysis and Generalized Differentiation. I: Basic Theory, Grundlehren der Mathematischen Wissenschaften [Fundamental Principles of Mathematical Sciences], vol. 330. Springer, Berlin (2006)

37. Mordukhovich, B.S., Ouyang, W.: Higher-order metric subregularity and its applications. Optimization Online (07/4440), 1-17 (2014)

38. Ngai, H.V., Théra, M.: Error bounds and implicit multifunction theorem in smooth Banach spaces and applications to optimization. Set-Valued Anal. 12(1-2), 195-223 (2004)

39. Ngai, H.V., Théra, M.: Error bounds in metric spaces and application to the perturbation stability of metric regularity. SIAM J. Optim. 19(1), 1-20 (2008)

40. Ngai, H.V., Tron, N.H., Théra, M.: Hölder metric subregularity via error bounds Preprint

41. Pang, J.S.: Error bounds in mathematical programming. Math. Programming, Ser. B 79(1-3), 299-332 (1997)

42. Penot, J.P.: Metric regularity, openness and Lipschitzian behavior of multifunctions. Nonlinear Anal. 13(6), 629-643 (1989)

43. Penot, J.P.: Error bounds, calmness and their applications in nonsmooth analysis. In Nonlinear analysis and optimization II. Optimization, Contemp. Math., vol. 514, pp. 225247. Amer. Math. Soc., Providence, RI (2010)

44. Penot, J.P.: Calculus without Derivatives, Graduate Texts in Mathematics, vol. 266. Springer, New York (2013)

45. Rockafellar, R.T., Wets, R.J.B.: Variational Analysis. Springer, Berlin (1998)

46. Yen, N.D., Yao, J.C., Kien, B.T.: Covering properties at positive-order rates of multifunctions and some related topics. J. Math. Anal. Appl. 338(1), 467-478 (2008)

47. Zălinescu, C.: Convex Analysis in General Vector Spaces. World Scientific Publishing Co. Inc., River Edge, NJ (2002)

48. Zheng, X.Y., Ng, K.F.: Metric subregularity and constraint qualifications for convex generalized equations in Banach spaces. SIAM J. Optim. 18, 437-460 (2007)

49. Zheng, X.Y., Ng, K.F.: Metric subregularity and calmness for nonconvex generalized equations in Banach spaces. SIAM J. Optim. 20(5), 2119-2136 (2010)

50. Zheng, X.Y., Ng, K.F.: Metric subregularity for proximal generalized equations in Hilbert spaces. Nonlinear Anal. 75(3), 1686-1699 (2012)

51. Zheng, X.Y., Ouyang, W.: Metric subregularity for composite-convex generalized equations in Banach spaces. Nonlinear Anal. 74(10), 3311-3323 (2011) 